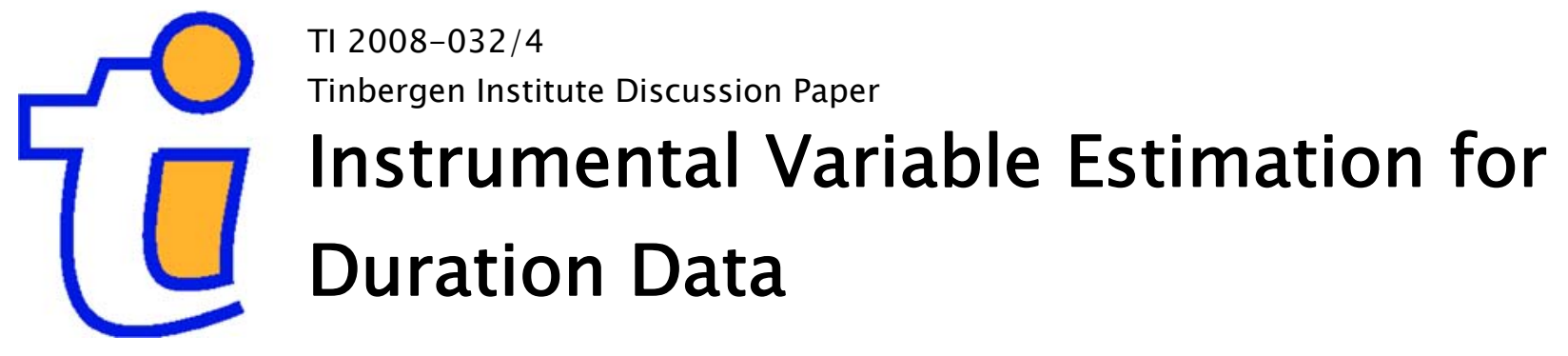

Govert E. Bijwaard

Econometric Institute, Erasmus University Rotterdam. 


\section{Tinbergen Institute}

The Tinbergen Institute is the institute for economic research of the Erasmus Universiteit Rotterdam, Universiteit van Amsterdam, and Vrije Universiteit Amsterdam.

Tinbergen Institute Amsterdam

Roetersstraat 31

1018 WB Amsterdam

The Netherlands

Tel.: $\quad+31(0) 205513500$

Fax: $\quad+31(0) 205513555$

Tinbergen Institute Rotterdam

Burg. Oudlaan 50

3062 PA Rotterdam

The Netherlands

Tel.: $\quad+31(0) 104088900$

Fax: $\quad+31(0) 104089031$

Most TI discussion papers can be downloaded at http:/ /www.tinbergen.nl. 


\title{
Instrumental Variable Estimation for Duration Data
}

\author{
Govert E. Bijwaard* \\ Econometric Institute \\ Erasmus University Rotterdam
}

\begin{abstract}
In this article we develop an Instrumental Variable estimation procedure that corrects for possible endogeneity of a variable in a duration model. We assume a Generalized Accelerated Failure Time (GAFT) model. This model is based on transforming the durations and assuming a distribution for these transformed durations. The GAFT model encompasses two competing approaches to duration data; the (Mixed) Proportional Hazard (MPH) model and the Accelerated Failure Time (AFT) model. The basis of the Instrumental Variable Linear Rank estimator (IVLR) is that for the true GAFT model the instrument does not influence the hazard of the transformed duration. The inverse of an extended rank test provide the estimation equations the IVLR estimation procedure is based on. We discuss the large sample properties and the efficiency of this estimator. We discuss the practical issues of implementation of the estimator.

We apply the IVLR estimation approach to the Illinois re-employment bonus experiment. In this experiment individuals who became unemployed were divided at random in three groups: two bonus groups and a control group. Those in the bonus groups could refuse to participate in the experiment. It is very likely that this decision is related to the unemployment duration. We use the IVLR estimator to obtain the effect of these endogenous claimant and employer bonuses on the re-employment hazard.
\end{abstract}

JEL classification: C21, C41, J64.

Key words: Endogenous Variable; Duration model; Censoring; Instrumental Variable.

*Erasmus University Econometric Institute, P.O. Box 1738, 3000 DR Rotterdam, The Netherlands; Phone: (+31) 1040 81424; Fax: (+31) 1040 89162; E-mail: bi jwaard@few.eur.nl. This research is financially supported by the Netherlands Organization for Scientific Research (NWO) nr. 451-04-011. I am very grateful to Geert Ridder for his comments and suggestions. I also benefited from the comments of participants at the 2003 Extending the Tinbergen Heritage conference in Rotterdam, at the 2003 ESEM Meeting, at the $14^{\text {th }}$ EC $^{2}$ conference, at the Econometric Evaluation of Public Policies : Methods and Applications in Paris 2005 and at the Causal Analysis in Population Studies conference in Vienna 2006 for comments and feedback. I thank Steve Woodbury and the W. E. Upjohn Institute for making the data available to me. 


\section{Introduction}

Social scientists have a long tradition of exploring the substantive implications of endogeneity in both methodological work and empirical work. Endogeneity is troublesome because it precludes the usual causal kinds of statements social scientists like to make. A canonical example is the evaluation of the effect of training programs of unemployment individuals on earnings and employment status. In general, the indicator for those who were trained is endogenous, because those individuals who choose to get training perceive the training as beneficial for earning or employment status. Other examples include the effect of union status and childbearing on labor market outcomes. All these problems have a treatment-control flavor. The notion that treatment status is endogenous reflects the fact that simple comparisons of treated and untreated individuals are unlikely to have a causal interpretation.

In recent years, social experiments have gained popularity as a method for evaluating social and labor market programs (see e.g. Meyer (1995), Heckman et al. (1999) and Angrist and Krueger (1999)). In experiments the assignment of individuals to the treatment can be manipulated. If assignment is random, the average impact of the treatment can be estimated. However, a randomized assignment may be compromised, if the individuals can refuse to participate, either by dropping out, if they are to receive the treatment, or by obtaining the treatment, if they are in the control group. If this non-compliance to the assigned treatment is correlated with the outcomes in the treatment or control regimes, the observed effect of the treatment is a biased estimate of the treatment effect. Thus, even with random assignment the actual treatment status can be endogenous.

Most of the evaluation literature has focused on static treatments, i.e. treatment that is administered at a particular point in time or in a particular time interval. If the outcome is a duration the treatment or its effect can be dynamic, i.e. it can be switched on and off over time. Examples are the unemployment insurance experiments (see Meyer (1995) for a survey) in which the unemployed receive a cash bonus if they find a job in a specified period. Another example is a temporary cut in unemployment benefits of unemployed individuals who do not expend sufficient effort to find a job (e.g. see Van den Berg et al. (2004) for The Netherlands, Lalive et al. (2005) for Switzerland and Ashenfelter et al. (2005) for the U.S. ).

The problem of endogeneity in duration models is similar to other statistical models: when endogeneity is present the standard interpretations given by any statistical model generally do 
not hold. If the training is perceived beneficial those individuals who choose to get training differ ex ante from those who choose not to get training. Similarly, unemployed who choose to be eligible for a cash bonus if they find a job in time, differ both in observed and unobserved characteristics that may influence their job finding probability. For linear models the problem of endogeneity can be solved if an instrument is available. The only requirement is that such an instrument affects the endogenous variable but is not correlated with the errors of the regression. We extend that notion to duration models that are inherently non-linear and propose an estimation technique.

In this article we assume the durations follow a Generalized Accelerated Failure Time (GAFT) model, a model introduced by Ridder (1990). The GAFT model is based on transforming the duration and assuming some distribution for this transformed duration. The transformation is related to the integrated hazard of a PH model. The AFT model is obtained by restricting the transformation. The AFT does not restrict the distribution of the transformed duration, while the MPH model restricts this distribution to a mixture of exponentials. The regression coefficients in a GAFT model can be interpreted in terms of the effect of regressing on the quantiles of the distribution of the transformed duration for the reference individual. In an AFT model the relation between the quantile of a individual with observed characteristics $X$ and the quantile of the reference individual is the acceleration factor. In a GAFT this acceleration factor is multiplied by the ratio of the 'duration dependence' at the two quantile durations.

The basis of the proposed Instrumental Variable Linear Rank estimator (IVLR) is that for the true GAFT model the instrument is independent of the transformed duration. The intuition behind this idea can be clarified by considering the simple example of a re-employment experiment with random assignment to treatment and a selective compliance. Assume that both the assignment and the compliance decision are made at the start of the study. If the treatment has no impact on the re-employment hazard, then the probability of observing an individual from the treatment group among those still unemployed at a given unemployment duration should be equal to the treatment assignment probability at the start. However, if the treatment has a positive effect on the hazard the probability of observing an individual from the treatment group among those still unemployed declines with the duration, because the treated individuals find a job faster. A GAFT model transforms the duration and for the true transformed durations the hazard of these transformed durations does not depend on the treatment group. This implies 
that the proportion of people in the treatment group on the transformed duration time remains the same, and is equal to the treatment assignment probability.

The IVLR estimation method uses the inverse of the rank test to obtain the parameters of the GAFT model, including the effect of the endogenous variable. The rank test is a commonly applied method to test the significance of a covariate on the hazard. The test is based on (possibly weighted) comparisons of the estimated non-parametric hazard rates. It is also equivalent to the score test for significance of a (vector of) coefficient(s) that arises from the Cox partial likelihood. The test rejects the influence of the covariate(s) on the hazard when it is 'close' to zero. Tsiatis (1990) shows that the inverse of the rank test can be used as an estimation equation for AFT models. The inverse of the rank test is the value of the (vector of) coefficient(s) that makes the rank-test equal to zero. Here we extend the inverse rank estimation to a GAFT model, which also includes the parameters of the transformation.

A common feature of duration data is that the durations are (right)-censored, the sense that we only know that their realisation exceeds the censoring time. The existence of endogenous covariates implies (possible) dependence between the transformed duration and the censoring time. This implies that the IVLR estimator, which exploits the independence between the transformed durations and the instruments, may give biased results. We can often make the assumption that the (potential) censoring time is known at the start of the study. In the reemployment bonus data, for example, we can only observed the unemployed while receiving UI benefits. In this case the potential censoring time for all individuals is at 26 weeks, the maximum duration of UI benefits in Illinois at the time of the experiment. With known (potential) censoring time we can modify the GAFT transformation by introducing additional censoring such that this modified transformation and the instruments become independent for the uncensored observations. Then, the IVLR estimator on this modified transformation leads to consistent estimators.

The IVLR estimation is based on a vector of mean restrictions on weight functions of the covariates, instrument and the transformed durations. Thus the IVLR is also related to GMM estimation. In GMM estimation it is feasible to get the most efficient GMM estimator in just two steps. In the first step directly observed weighting matrices lead to a consistent, but not necessary efficient estimator. From this consistent estimator we can consistently estimate the efficient weighting matrices. It is then possible to obtain an efficient estimate of the parameters involved in just one additional step. A similar reasoning applies to the IVLR-estimator. In the 
first step we use simple weighting functions to obtain consistent estimates of the parameters of the GAFT model. From these parameters we can estimate the distribution of the transformed durations, which are needed to calculate the most efficient weighting functions. Then, in just one additional step the efficient IVLR is obtained.

For our empirical application we use data from the Illinois unemployment bonus experiment. These data have been analysed before with increasing sophistication by Woodbury and Spiegelman (1987), Meyer (1996) and Bijwaard and Ridder (2005). In this experiment a group of individuals who became unemployed during four months in 1984 were divided at random in three groups of about equal size: two bonus groups and a control group. The unemployed in the claimant bonus group qualified for a cash bonus if they found a job within 11 weeks and would hold this job for at least four months. In the employer bonus group, the bonus was paid to their employer. The members of the two bonus groups were asked whether they were prepared to participate in the experiment. About $15 \%$ of the claimant bonus and $35 \%$ of the employer bonus groups refused participation. It is very likely that the decision to be eligible for a bonus is related to the unemployment duration. This makes the participation indicator an endogenous variable in relation to the unemployment duration.

The outline of the article is as follows. Section 2 discusses the problems associated with endogenous variables in duration models. We introduce the GAFT model and discuss the interpretation of the parameters of a GAFT model. We also give the intuition for the idea that transforming of the durations, inherent in the GAFT model, provide the basis for estimating the effect of endogenous covarites. In Section 3 we introduce the IVLR estimator, derive its asymptotic properties and discuss the efficiency and the practical implementation of the estimator. Section 4 discusses the empirical application of the IVLR estimator to the re-employment bonus experiment. We conclude with a summary and discuss possible avenues for further research in Section 5.

\section{Endogenous Covariates in Duration Models}

For many economic and demographic phenomena the timing of a transition from one state into another state is important. Examples include the time till re-employment of an unemployed individual, the time till marriage and the time till death. Two important features of such transition data are that relevant characteristics of the individual may change over time and that, due to a limited observation window, we do not observe the completed duration for 
all individuals. In a duration model the timing of a particular event is modeled and it is straightforward to incorporate time-varying variables and allow for (right)-censoring.

The key variables in duration analysis are the duration till the next event, $T$, and the indicator of censoring, $\delta$. The observed durations may be right-censored, i.e. we observe $\tilde{T}=$ $\min (T, C)$ with $C$ the censoring time. The possible time-varying covariates are given by the vector $X_{i}(t)$ where $i$ refers to a member of the population. The path of the covariates are predetermined. Thus $\bar{X}(t)=\{X(s) ; 0 \leq s \leq t\}$ does not depend on future events.

Two competing approaches for the analysis of duration data has been the (Mixed) Proportional Hazard (MPH) model and the Accelerated Failure Time (AFT) model. The MPH model assumes that the covariates and the unobserved heterogeneity affect the baseline hazard proportionally (see Van den Berg (2001) for a recent overview). The AFT model assumes that the covariates affect the duration proportionally. An AFT model implies that the distribution of the duration of an individual with covariate vector $X$ and the transformed duration distribution

of $e^{-\beta^{\prime} X} T$ are the same (see a.o. Brännäs (1992), Kalbfleisch and Prentice (2002)). Thus the covariate accelerates the duration, when the coefficient $\beta$ is smaller than zero, or decelerates the duration, when the coefficient is greater than zero. This is equivalent to a linear regression model for the log-duration.

\subsection{The Generalized Accelerated Failure Time Model}

A class of duration models that generalizes the AFT models in such a way that it also includes the MPH models is the Generalized Accelerated Failure Time (GAFT) model. The GAFT model, , introduced by Ridder (1990), is not specified by the distribution of the logduration. Instead, we transform the duration, and assume that this transformed duration has some distribution, either known or unknown. The transformation of the duration is related to the integrated hazard in a PH-model. The GAFT model is also related to the generalized regression model proposed by Han (1987).

The GAFT model assumes that the relation between the duration $T$ and the covariates is specified as

$$
\int_{0}^{T} \lambda(s ; \alpha) e^{\beta^{\prime} X(s)} \mathrm{d} s=U
$$

where $\lambda(t ; \alpha)$ is a non-negative 'baseline' function on $[0, \infty)$. In the sequel we assume that $\lambda$ is 
the piecewise constant function, i.e.

$$
\lambda(t, \alpha)=\sum_{j=0}^{J} e^{\alpha_{j}} I\left(t_{j}<t \leq t_{j+1}\right)
$$

with $t_{0}=0$ and $t_{L+1}=\infty$ and the hazard on the last interval is normalized to $1, \alpha_{L}=0$. Other $\lambda$-functions are also possible. The non-negative regression function $e^{\beta^{\prime} X(s)}$ captures the effect of the covariates.

The GAFT model is characterized by these baseline and regression functions and by the distribution of the non-negative random variable $U$. We denote the survivor function of $U_{0}$, the transformation in the true population parameters $\alpha_{0}$ and $\beta_{0}$, by $\bar{G}_{0}(u)$ and its hazard function by $\kappa_{0}(u)$. We assume that the distribution of $U_{0}$ is absolutely continuous and independent of $X$. The semi-parametric estimators considered in this article avoid assumptions on the distribution of $U_{0} \cdot 1$

As mentioned, the GAFT model contains as special cases the AFT, the PH and the MPH models. The AFT model restricts the transformation to $\lambda(t ; \alpha) \equiv 1$, but leaves the distribution of $U_{0}$ unrestricted (with the exception of that $U_{0}$ should be non-negative, see e.g. Cox and Oakes (1984)). The (M)PH model restricts the distribution of $U_{0}$, but leaves the $\lambda$ unrestricted (non-negative). The distribution of $U_{0}$ is an unit exponential distribution $(\mathrm{PH})$ or a mixture of exponential distributions (MPH).

We can interpret the GAFT model in terms of the effect of regressing on baseline quantiles, the quantiles for the reference individual. To illustrate this let $t_{q}(\bar{X})$ be the $q$-th quantile of the distribution of duration with covariate history $\bar{X}$. Let $t_{q}$ be the $q$-th quantile for the reference individual (i.e. with $X(t)$ identically equal to zero). Then the ratio of the change in quantiles is

$$
\frac{d t_{q}(\bar{X})}{d t_{q}}=e^{-\beta_{0}^{\prime} X\left(t_{q}(\bar{X})\right)} \frac{\lambda\left(t_{q} ; \alpha_{0}\right)}{\lambda\left(t_{q}(\bar{X}) ; \alpha_{0}\right)}
$$

In an AFT model the ratio of the quantiles is the acceleration factor $e^{-\beta_{0}^{\prime} X}$. Thus, in the GAFT model the ratio of the change in the quantiles is the acceleration factor multiplied by the ratio of the values of the baseline $\lambda(t)$ evaluated at the $q$-th quantile of the reference duration and the $q$-th quantile of the duration with covariate $X$.

In the MPH model we can interpret $\lambda(t)$ as the baseline hazard, i.e. the factor in the proportional hazard that captures the (duration) time variation in the hazard function. Thus, in the MPH model the ratio in (3) can be interpreted as the ratio of baseline hazards. The

\footnotetext{
${ }^{1}$ In appendix A we show when the parameters of GAFT model are identified.
} 
regression parameter, $\beta$, is the proportional change in the hazard rate due to a unit change in $X(t)$ for a unit with unobserved heterogeneity $V$.

\subsection{Endogenous Covariates in GAFT Models}

It can rarely be defended that a study on unemployment durations includes all the relevant characteristics of the individuals looking for a job. For example, consider our application of analysing the effect of a cash-bonus on the re-employment probability. Because such a bonus increases the reward of leaving unemployment it gives an incentive to search more intensively and therefore it increases the re-employment hazard. However, the search intensity of the unemployed individuals is usually not observed. Suppose that the unemployed have to choose at the start of their unemployment spell whether they want to be eligible for a bonus. If they choose to be eligible they have to fill in some forms, notify their new employer and provide a proof that they held that new job for at least four months. Thus, joining the bonus program implies some administrative duties for the unemployed and cooperation with their new employer. This might refrain some individuals from joining the bonus program. It is very likely that the unobserved motivation to return to work has an impact on both the decision to join the bonus program and the search intensity. This implies that the indicator of joining the bonus program is an endogenous variable for the analysis of the unemployed duration. Without adjusting for this (self)-selection standard duration analysis give biased results of the effect of the bonus on unemployment duration.

A way of adjusting for an endogenous variable is the conventional instrumental variable method that assumes instrument-error independence and an exclusion restriction. A familiar example of an instrumental variable is the treatment assignment-indicator of a randomly assigned treatment in which the actual treatment still depends on a decision by the agents (or on decisions made by those who execute the program). For instance, long-term unemployed can be randomly assigned to a training program, but for many programs they can still decide not to join, or the training manager can decide to withhold some training from some people. Then, the assignment indicator is an instrument for the actual indicator of training received.

The method of instrumental variables (IV) is widely used in econometrics. For illustration consider the simple linear model

$$
Y=\beta^{\prime} X+\gamma D+\epsilon
$$

where $Y$ is observed outcome, $X$ is a vector of exogenous variables, $D$ is an endogenous variable, 
and $\epsilon$ is a disturbance with mean 0 . If $D$ and $\epsilon$ are correlated OLS gives biased estimates of $\theta=(\beta, \gamma)$. The conventional IV method uses an instrument $R$ that affects $D$ but is uncorrelated with $\epsilon$, like the assignment indicator in a random but compromised experiment. If we denote $Z=(X, R)$ and $\tilde{X}=(X, D)$ the IV estimator is

$$
\hat{\theta}_{I V}=\left(Z^{\prime} \tilde{X}\right)^{-1} Z^{\prime} Y
$$

Complications arise if the outcome variable of interest is a duration variable, like the unemployment duration. Models for duration data are usually non-linear in the mean. Then the standard IV-methods can not be applied. An important issue in duration models ia that the value of the endogenous variable may depend on information that accumulates during the evolution of the duration. The common approach to accommodate such time-varying variables is to relate them to the hazard rate. Another issue is that duration data are usually (right)-censored, due to a limited observation window. The hazard rate is invariant to censoring and is therefore the natural choice for the analysis of duration data.

In this paper we provide an instrumental variable method for duration data based on inference on the hazard rate. Let $D(t)$ be the value of the endogenous variable at duration $t$. The GAFT model with endogenous variables is

$$
\int_{0}^{T} \lambda(s ; \alpha) \exp \left(\beta^{\prime} X(s)+\psi(s, D(s), \gamma)\right) \mathrm{d} s=U=h(T, \bar{X}(T), \bar{D}(T), \theta)
$$

where $\psi(t, D(t), \gamma)$ captures the effect of the endogenous variable and $\theta=\left(\beta^{\prime}, \alpha^{\prime}, \gamma^{\prime}\right)^{\prime}$ is the whole parameter vector. Without loss of generality we assume that the endogenous variable is binary and only changes at prediscribed durations. We also assume that the effect of the endogenous variable may change over the duration. Then a flexible functional form for the 'treatment' function is

$$
\psi(t, D(t), \gamma)=\sum_{j=0}^{J} \gamma_{j} \cdot D_{j} \cdot I_{j}(t)
$$

where $I_{j}(t)=I\left(t_{j}<t \leq t_{j+1}\right)$ are interval indicators with $t_{0}=0$ and $t_{J+1}=\infty$.

If $D$ were exogenous, standard techniques for the analysis of survival time data could be used to estimate the $\gamma$ 's. For example, we can use a Mixed Proportional Hazards model and estimate $\gamma$ using (semi-parametric) Maximum Likelihood procedures, depending on the assumptions we make about the distribution of the unobserved heterogeneity, $V$, and the baseline hazard. If the model is correctly specified the MLE yields a consistent estimate. However, we will get biased 
estimation results for the parameters if the covariate is endogenous. The problem is that those who comply with their assigned treatment differ in observed and unobserved characteristics from those who do not comply.

Since physical randomization implies that at time zero all attributes of the two treatment groups are (in expectation) identical, a commonly used solution to this problem is to ignore the post-randomization compliance and rely on the analysis of the treatment assignment groups. This intention-to-treat (ITT) analysis replaces the actual value of the endogenous variable, $D$ by the instrument, $R$ in the estimation procedure. Further, if the model is correctly specified the estimated $\gamma$ 's effect will correspond to the overall effect that would be realized in the whole population, under the assumption that the compliance rate and the factors influencing compliance in the sample are identical to those that would occur in the whole population.

The major drawback of the intention-to-treat analysis is that the estimated effect is a mixture of the population effect and the effect on the compliance. Hence, if the treatment effectively raises the re-employment hazard, the intention-to-treat measure of this effect will diminish as non-compliance increases. Another disadvantage is that compliance is very likely to depend on the perceived effects of the treatment. If, for example, the unemployed know that being eligible for a re-employment bonus does not stigmatize them, they will be more prone to participate. Thus, when the pattern of compliance is a function of the perceived efficacy of the treatment the estimated intention-to-treat will not represent the overall effect of the treatment had it been adopted in the whole population.

\subsection{Intuition for Instrumental Variable estimation}

The basis of the proposed Instrumental Variable Linear Rank estimator (IVLR) is that for the true GAFT model the instrument is independent of the transformed duration. This implies that the proportion of people in the treatment group, $R=1$ on the (true) transformed duration time remains the same, and is equal to the treatment assignment probability. Thus, for the true transformed duration $U_{0}=h\left(T, \bar{X}(T), \bar{D}(T), \theta_{0}\right)$ we have

$$
\operatorname{Pr}\left(R=1 \mid U_{0} \geq u\right)=\operatorname{Pr}\left(R=1 \mid U_{0}=0\right)
$$

This implies that the hazard of the true transformed duration is independent of the instrument. This independence only holds for the true parameters and we can therefore build an estimation procedure that exploits this conditional independence. In the next section we introduce our 
proposed method based on this condition independence assumption. ${ }^{2}$. First we discuss the implications of right-censoring on these independence assumption.

A common feature of duration data is that some of the observations are censored. Assume the censoring time, $C$, is (potentially) known. For example, in the analysis of unemployment duration based on administrative data the duration is often only observed while the individual receives unemployment benefits. Usually, the maximum duration of receiving benefits is based on the labor market history of the individual and is recorded in the data. Then, the potential censoring time is known and the observed durations are $\tilde{T}=\min (T, C)$ and $\Delta=I(T \leq C)$, where $\Delta$ is one if $T$ is observed.

One is tempted to define the censored transformed durations by the minimum of the transformed time till (potential) censoring and the transformed time till the event occurs, $\tilde{U}(\theta)=\min (h(T ; \theta), h(C ; \theta))=h(\tilde{T} ; \theta)$. However, the existence of endogenous covariates and censoring makes some of the orthogonality conditions fail to hold. This can be illustrated by a simple example: Consider a fixed censoring time, all individuals have the same maximum duration of receiving benefits. Then for all individuals, irrespective of their value of the endogenous variable, censoring occurs at time $C$. Suppose the binary endogenous variable, $D$, and other covariates all be determined at the start of the study and have a constant effect on the hazard. Finally, we assume that except for $\gamma$ the effect of the endogenous variable, all parameters, $\beta_{0}$ and $\alpha_{0}$, are known. Then, the transformation is

$$
U_{0}=e^{\gamma_{0} D+\beta_{0}^{\prime} X} \Lambda_{0}(T)
$$

with $\Lambda_{0}(t)=\int_{0}^{t} \lambda\left(s, \alpha_{0}\right) \mathrm{d} s$. Hence, if $D=0$ censoring in the transformed time occurs at $e^{\beta_{0}^{\prime} X} \Lambda_{0}(C)$, but if $D=1$ censoring occurs at $e^{\beta_{0}^{\prime} X+\gamma_{0}} \Lambda_{0}(C)$. Thus, if $\gamma_{0}>0$, then all transformed durations in the interval $\left[e^{\beta_{0}^{\prime} X} \Lambda_{0}(C), e^{\beta_{0}^{\prime} X+\gamma_{0}} \Lambda_{0}(C)\right]$ have $D=1$ (for $\gamma_{0}<0$ the boundaries are reversed). The hazard of $U_{0}$ on this interval clearly depends on $D$ and hence on $R$. The independence of the hazard of $U_{0}$ and $R$ only holds up to the lower bound of the interval. This implies that in the IVLR, which exploits this independence, the transformed durations that fall in the problematic interval have to be censored. In Appendix B we derive the additional censoring required in a more general setting. This additional censoring, $C^{U}(\theta)$, depends on the (unknown) parameters. The IVLR estimation method is than based on the

\footnotetext{
${ }^{2}$ Here we only concentrate on a static binary instrument and a discrete, but possible time-varying according to a prescribed protocol, endogenous variable. It is not difficult to extend the analysis to more, discrete, levels of both the instrument and the endogenous variable and to have a sequential instruments.
} 
(transformed) durations $\tilde{U}(\theta)=\min \left(U(\theta), C^{U}(\theta)\right)$, with $U(\theta)$ given in (4) and the censoring indicator $\Delta^{U}(\theta)=I\left(U(\theta)<C^{U}(\theta)\right)$. Then for the 'uncensored' observations, that is for $\Delta^{U}(\theta)=1$, the transformed duration $\tilde{U}(\theta)$ is independent of the instrument. This is explained in more detail in Appendix B.

\section{Instrumental Variable Linear Rank Estimation}

In this section we introduce an Instrumental Variable method for duration models that adjusts for the possible endogeneity of the intervention, without suffering the problems of the intentionto-treat method. The basis of this IVLR estimator is that for the true GAFT model the instrument does not influence the hazard of the transformed duration. A typical way to test the significance of a covariate is the rank-test, see Prentice (1978). The test is based on (possibly weighted) comparisons of the estimated non-parametric hazard rates. It is also equivalent to the score test for significance of a (vector of) coefficient(s) that arises from the Cox partial likelihood. The test rejects the influence of the covariate(s) on the hazard when it is 'close' to zero. Tsiatis (1990) shows that the inverse of the rank test can be used as an estimation equation for AFT models. The inverse of the rank test is the value of the (vector of) coefficient(s) that puts the rank-test equal to zero. Here we extend the inverse rank estimation to a GAFT model, which also includes the parameters of the duration dependence.

\subsection{The IVLR estimator}

Before we turn to the general model we discuss a simple AFT example to provide more insight into the inverse rank estimation approach. Suppose we would like to test whether a covariate $X$ influences the hazard. If the covariate does not influence the hazard, the mean of the covariate among the survivors does not change with the survival time, i.e. $\mathrm{E}[X \mid T \geq t]=\mathrm{E}[X]$. Define the observation indicator, that is the indicator that individual $j$ is still alive (unemployed) at time $t$, by $Y_{i}(t)=I\left(t_{i} \geq t\right)$. Then the rank test-statistic is (assuming no censoring)

$$
\sum_{i}^{n}\left[X_{i}-\frac{\sum_{j} Y_{j}\left(t_{i}\right) X_{j}}{\sum_{j} Y_{j}\left(t_{i}\right)}\right]
$$

where the second term is the mean of the covariate among those individuals still alive at $t_{i}$. Thus for each observation of the covariate we compare the observed value with its expected value among those still alive (under the hypothesis of no effect of the covariate) and sum over all observations. If this sum is significantly different from zero, we reject the null of no influence. 
Now assume that the true model is an AFT-model with $U=e^{\beta X} T$. Then, for the true parameter $\beta=\beta_{0}$ the hazard of $U$ does not depend on the covariate $X$. This implies that the rank statistic for the true parameter on the transformed $U$-time is zero. However the $\beta_{0}$ is unknown and an inverse rank estimate $\hat{\beta}$ of $\beta_{0}$ is the value of $\beta$ for which

$$
\sum_{i}^{n}\left[X_{i}-\frac{\sum_{j} Y_{j}^{U}\left(U_{i}\right) X_{j}}{\sum_{j} Y_{j}^{U}\left(U_{i}\right)}\right]=0
$$

with $U_{i}=e^{\hat{\beta} X_{i}} t_{i}$ and $Y_{j}^{U}(u)=I\left(U_{j} \geq u\right)$, the observation indicator on the (transformed) $U$-time. Tsiatis (1990) derives the asymptotic properties of this estimator. Robins and Tsiatis (1991) discuss how the rank estimator can be used to estimate the effect of an endogenous variable in an AFT-model.

We extend the method of Robins and Tsiatis (1991) to GAFT models. We use the transformed GAFT durations in (4) and adjust them for censoring, see Appendix B. Just as in the example above, we have that for the population parameter vector $\theta_{0}$ the hazard of the implied transformed duration $U_{0}$, which is $\kappa_{0}(u)$, is independent of the covariate and instrument history up to $h_{0}^{-1}(u)$. Because this is true only for $\theta=\theta_{0}$, we can use the inverse of the rank statistic to get an estimate of $\theta_{0}$. Note that for notational convenience we suppress the dependence on $\theta$ in censored durations $\tilde{U}(\theta)$.

The estimating equations that defines the IVLR estimator contain a left-continuous vector weight function $W$. The weight function may depend on $\tilde{U}_{i}(\theta)=\tilde{U}_{i}, \bar{X}_{i}^{U}(u)$ and $R$. Typical examples are $W=\left(W_{\beta}, W_{\gamma}, W_{\alpha}\right)$ with $W_{\beta}=X$ for the coefficient vector $\beta$ of the exogenous variables and $W_{\gamma}=R$, the instrument, for a dummy endogenous variable $D$ and $W_{\alpha_{j}}=I_{j}(u)=$ $I\left(h\left(t_{j}\right)<u \leq h\left(t_{j+1}\right)\right)$ for a piecewise constant baseline hazard on intervals $\left(t_{j}, t_{j+1}\right]$. The variance of the IVLR estimator depends on the choice of the weight-function and in section 3.2 we discuss the optimal choice of this function. For a given choice of the weight-function and possible additional censoring the IVLR estimator is defined by the estimating equations

$$
\left.S_{n}(\theta ; W)=\sum_{i=1}^{n} \Delta_{i}^{U}\left\{W\left(\tilde{U}_{i}, \bar{X}_{i}^{U}\left(\tilde{U}_{i}\right), R_{i} ; \theta\right)\right)-\bar{W}\left(\tilde{U}_{i} ; \theta\right)\right\}
$$

where

$$
\bar{W}\left(\tilde{U}_{i} ; \theta\right)=\frac{\left.\sum_{j=1}^{n} Y_{j}^{U}\left(\tilde{U}_{i}\right) W\left(\tilde{U}_{i}, \bar{X}_{j}^{U}\left(\tilde{U}_{i}\right), R_{j} ; \theta\right)\right)}{\sum_{j=1}^{n} Y_{j}^{U}\left(\tilde{U}_{i}\right)}
$$

is the average weight function among the individuals still at risk at the transformed duration 
$\tilde{U}_{i}(\theta)$. Note that we use $\Delta_{i}^{U}$ instead of $\Delta_{i}$ to assure independence of the instruments and the transformed durations for all uncensored observations.

The interpretation of the estimation equations is that it compares the value of the weight function at a transformed duration $\tilde{U}_{i}(\theta)$ to the average of the weight functions for those individuals that are still at risk at that particular transformed duration. For the true parameter vector $\theta_{0}=\left(\beta_{0}, \alpha_{0}, \gamma_{0}\right)$ the expected difference of the weight function and its average for those still at risk is zero. Thus, the statistic $S_{n}(\theta ; W)$ has mean zero for the true parameters. We therefore base our estimator on the roots of $S_{n}(\theta ; W)=0$, which is the inverse of the extended rank statistic. However, the estimating functions are discontinuous, piecewise constant, functions of $\theta$ and a solution may not exist. For that reason we define the Instrumental Linear Rank estimator (IVLR) $\hat{\theta}_{n}(W)$ as the minimizer of the quadratic form, i.e.

$$
\hat{\theta}_{n}(W)=\inf \left\{\theta \mid S_{n}(\theta ; W)^{\prime} S_{n}(\theta ; W)\right\}
$$

To ensure weak consistency and asymptotic normality of the IVLR estimator we make the following assumptions. The random variable $R$ is an instrument that is determined at the start. We restrict both the instrument, $R$, and the endogenous variable $D$, to be binary. The other assumptions can be found in appendix C.

If $S_{n}(\theta ; W)$ were differentiable with respect to $\theta$, then asymptotic normality can be proved using Taylor series expansion in a neighborhood of $\theta_{0}$. Tsiatis $(1990)$ showed that, if $S_{n}(\theta ; W)$ is not differentiable, as in the current problem, we can still use a linear approximation of $n^{-1 / 2} S_{n}(\theta ; W)$. Using this approximation and the asymptotic normality of $S_{n}\left(\theta_{0} ; W\right)$, we can show that $\sqrt{n}\left(\hat{\theta}_{n}(W)-\theta_{0}\right)$ is asymptotically normal. For the derivation of the asymptotic properties we use counting process theory (see Appendix B). Let $a\left(u ; \theta_{0}\right)$ be the probability limit of the average weight function (see assumption A6), $C_{0}$ the transformed censoring time for $\theta=\theta_{0}$. Let $d_{i 0}(u)$ the derivative of the hazard of $U(\theta)$ w.r.t. $\theta$, i.e.

$$
d_{i 0}(u)=\left.\frac{\partial \kappa_{i}^{U}(u ; \theta)}{\partial \theta}\right|_{\theta=\theta_{0}}
$$

and $V(u, \theta)$ is the probability limit of

$$
\frac{1}{n} \sum_{i=1}^{n}\left[W\left(u, \bar{X}_{i}^{U}(u), R_{i}\right)-\bar{W}(u ; \theta)\right] \times d_{i 0}(u)^{\prime} Y_{i}^{U}(u)
$$

The asymptotic properties of the IVLR estimator are summarized in the following two theorems. 
Theorem 1 (Consistency).

If assumptions $C 1$ to $C 7$ hold $\hat{\theta}_{n}(W)$ converges in probability to $\theta_{0}$.

Proof: See Appendix C.

Theorem 2 (Asymptotic Normality).

If assumptions $C 1$ to $C 9$ hold and $Q(W)$ has full rank, then

$$
\sqrt{n}\left(\hat{\theta}_{n}(W)-\theta_{0}\right) \stackrel{d}{\rightarrow} N\left(0, Q^{-1}(W) \Omega(W) Q^{-1}(W)\right)
$$

where

$$
\Omega(W)=\int_{0}^{C_{0}} a\left(u ; \theta_{0}\right) \kappa_{0}(u) d u
$$

is the asymptotic variance of $n^{-1 / 2} S_{n}\left(\theta_{0} ; W\right)$ and,

$$
Q(W)=\int_{0}^{C_{0}} V\left(u, \theta_{0}\right) d u
$$

the limiting covariance matrix of the processes $W\left(u, \bar{X}_{i 0}(u), R_{i}\right)$ and $d_{i 0}(u) / \kappa_{0}(u)$.

Proof: See Appendix C.

\subsection{Efficiency of the IVLR estimator}

Many different choices of the weight functions lead to consistent estimates of the parameters. By properly choosing the weight function the asymptotic variance of the IVLR can be minimized. Tsiatis (1990) has shown that for the AFT model with exogenous covariates weight functions proportional to $u \kappa_{0}^{\prime}(u) / \kappa_{0}(u) X$, with $\kappa_{0}(u)$ is the hazard of the true transformed durations $U_{0}$, minimize the asymptotic variance of the estimated regression parameters. In general the distribution of the true transformed duration, $U_{0}$, is unknown. This distribution can consistently be estimated from the implied transformed durations induced by IVLR-estimation with a weight function that does not depend on the transformed durations.

The IVLR estimation is based on a vector of mean restrictions on weight functions of the covariates, the instrument and the transformed durations. GMM estimation is also based on moment conditions and in GMM estimation it is feasible to get the most efficient GMM estimator in just two steps. A similar reasoning applies to the IVLR-estimator. This justifies an adaptive construction of an efficient estimator. In the next section we address the practical implementation of an adaptive estimation procedure. First, we introduce the optimal weight function. 
Theorem 3 (Optimal weight function in IVLR).

The weight-function that gives the smallest asymptotic variance for $\hat{\theta}_{n}(W)$ is

$$
\left.W_{\text {opt }}(u, \bar{X}(u), R) \propto \frac{\partial \ln \kappa^{U}(u ; \theta)}{\partial \theta}\right|_{\theta=\theta_{0}}=\frac{d_{i 0}(u)}{\kappa_{0}(u)}
$$

The asymptotic covariance matrix of the optimal IVLR estimator reduces to

$$
\Omega^{-1}\left(W_{\mathrm{opt}}\right)=Q^{-1}\left(W_{\mathrm{opt}}\right) .
$$

Proof of theorem 3. From

$$
\frac{1}{\sqrt{n}}\left(\begin{array}{c}
S_{n}\left(\vartheta_{0} ; W\right) \\
S_{n}\left(\vartheta_{0} ; W_{\mathrm{opt}}\right)
\end{array}\right) \stackrel{D}{\rightarrow} N\left(0,\left(\begin{array}{cc}
\Omega(W) & Q(W)^{\prime} \\
Q(W) & \Omega\left(W_{\mathrm{opt}}\right)
\end{array}\right)\right)
$$

follows that the matrix

$$
Z=\left(\begin{array}{cc}
\Omega(W) & Q(W)^{\prime} \\
Q(W) & \Omega\left(W_{\mathrm{opt}}\right)
\end{array}\right)
$$

is non-negative definite, the same is true for its inverse. In particular, the submatrices on the main diagonal of the inverse are non-negative definite. Hence the matrix

$$
Q^{-1}(W) \Omega(W) Q^{\prime-1}(W)-\Omega^{-1}\left(W_{\mathrm{opt}}\right)
$$

is a non-negative definite matrix.

Consider, for example, a GAFT model with a piecewise constant $\lambda$ function as defined in (2). Assume that the model has a constant coefficient for the endogenous variable then by (13) the optimal weight functions are

$$
\begin{aligned}
W_{\mathrm{opt}, \beta}= & X(u)\left[1+u \frac{\kappa_{0}^{\prime}(u)}{\kappa_{0}(u)}\right] \\
W_{\mathrm{opt}, \alpha_{\mathrm{j}}}= & \left(1+u \frac{\kappa_{0}^{\prime}(u)}{\kappa_{0}(u)}\right) \cdot\left(R I_{j}^{1}(u)+(1-R) I_{j}^{0}(u)\right)+ \\
& +R\left[\left(1+u \kappa_{0}(u)\right) \frac{f_{0}(u \mid 1, R)-f_{0}(u)}{f_{0}(u)}+u \frac{f_{0}^{\prime}(u \mid 1, R)-f_{0}(u)}{f_{0}(u)}\right] I_{j}^{1}(u)+ \\
& +(1-R)\left[\left(1+u \kappa_{0}(u)\right) \frac{f_{0}(u \mid 0, R)-f_{0}(u)}{f_{0}(u)}+u \frac{f_{0}^{\prime}(u \mid 0, R)-f_{0}(u)}{f_{0}(u)}\right] I_{j}^{0}(u) \\
W_{\mathrm{opt}, \gamma}= & R\left[1+u \frac{\kappa_{0}^{\prime}(u)}{\kappa_{0}(u)}\right]+ \\
& +R\left[\left(1+u \kappa_{0}(u)\right) \frac{f_{0}(u \mid 1, R)-f_{0}(u)}{f_{0}(u)}+u \frac{f_{0}^{\prime}(u \mid 1, R)-f_{0}(u)}{f_{0}(u)}\right]
\end{aligned}
$$

where $f_{0}(u \mid D, R)$ is the density of $U_{0}$ given $D$ and $R, f_{0}^{\prime}(\cdot)$ is the derivative of the density and $I_{j}^{D}(u)=I\left(m_{j}(X, D)<u \leq m_{j+1}(X, D)\right)$ for

$$
m_{j}(X, D)=\int_{0}^{t_{j}} \lambda(s, \alpha) e^{\beta^{\prime} X(s)+\gamma D} \mathrm{~d} s
$$




\subsection{Estimation in practice}

The statistic $S_{n}(\theta ; W)$ is a multi-dimensional step-function. Therefore, the standard NewtonRaphson algorithm cannot be used to solve the minimizer of the quadratic form of the estimation equations in (9). One of the alternative methods for finding the roots of a non-differentiable function is the Powell-method. This method (see Press et al. (1986, §10.5) and Powell (1964)) is a multidimensional version of the Brent algorithm. ${ }^{3}$

An additional difficulty in solving the estimation equations is that the (optimal) weightfunctions may depend on the, unknown, distribution of $U_{0}$. However, a consistent first stage estimator based on weight-functions that are independent of the distribution of $U_{0}$ is easy to find. For example, in a GAFT model with a piecewise constant $\lambda$ and a time-invariant coefficient of the endogenous variable, the choice for the first-step weight functions could be: $W=\left(X, R, I_{1}(u), \ldots, I_{J-1}(u)\right)$, with $X$ is the weight-function for the effect of the exogenous covariates, $R$ is the weight-function for the (time-constant) endogenous variable and, $I_{1}(u), \ldots, I_{J-1}(u)$ are the weight-functions for the parameters of the piecewise constant baseline hazard. Then based on the first stage estimator we can calculate the optimal weight functions. ${ }^{4}$

Related to the computation of optimal weight function is the estimation of the variance matrix for an arbitrary weight function. ${ }^{5}$ The difficulty in estimating the covariance matrix lies in the calculation of the matrix $Q(W)$ and not in the calculation of the variance matrix of the estimating equation. The latter can be consistently estimated by

$$
\hat{\Omega}=\frac{1}{n} \sum_{i=1}^{n} \Delta_{i}^{\hat{U}}\left[W\left(u, \bar{X}_{i}^{\hat{U}}(u), R_{i}\right)-\bar{W}(u, \hat{\theta})\right]\left[W\left(u, \bar{X}_{i}^{\hat{U}}(u), R_{i}\right)-\bar{W}(u, \hat{\theta})\right]^{\prime}
$$

where $\hat{U}$ is the value of $U(\hat{\theta})$.

Thus, the optimal weight functions, the covariance matrix and the most efficient estimators are estimated in two steps. The first step consists of obtaining a consistent estimate of $\theta_{0}$ using a weight function that does not depend on the distribution of $U_{0}$. The second step concerns the estimation of the unknown distribution of $U_{0}$, based on the transformed durations implied by the first step estimates. Many different methods are available to get a reasonable estimate of an unknown distribution. We shall not apply the commonly used kernel based

\footnotetext{
${ }^{3}$ See the site of Bo Honore http://www.princeton.edu/honore/ for the Powell method in Gauss.

${ }^{4}$ The estimation procedures written in Gauss are available upon request from the author.

${ }^{5}$ Robins and Tsiatis (1991) suggested to use a numerical derivative of $n^{-1} S_{n}(\theta ; W)$ that does not need an estimate of the optimal $W$-function to get $\hat{Q}(W)$. This numerical derivative is sensitive to the choice of the difference in $\theta$. We found it hard to get stable results.
} 
method. Although kernel-smoothed hazard rate estimators have been developed and adjusted to deal with the boundary problems inherent to hazard rates these methods can be difficult to implement due to the choice of the bandwidth. It is also unclear how the boundary corrections can be incorporated in the kernel estimates of the derivative of the hazard. We therefore choose to use a series approximation of the distribution.

Suppose the distribution of $U_{0}$ can be approximated arbitrary well using orthonormal polynomials. We base our approximation on Hermite polynomials using the exponential distribution as a weighting function:

$$
g_{0}(u)=\frac{a e^{-a u}}{\sum_{l=0}^{L} b_{l}^{2}}\left[\sum_{l=0}^{L} b_{l} L_{l}(u)\right]^{2}
$$

where

$$
L_{l}(u)=\sum_{k=0}^{l}\left(\begin{array}{l}
l \\
k
\end{array}\right) \frac{(-a u)^{k}}{k !}
$$

are the Laguerre polynomials. The unknown parameters of this approximation are $a$ and $b_{0}, \ldots, b_{L}$. If $b_{l} \equiv 0$ for all $l>0$ the distribution of $U_{0}$ is exponential. Even for $L$ as small as three (19) allows for many different shapes of $\kappa_{0}(u)$ and its derivative. Both can be derived analytically given the estimates of the parameters. The parameter estimators can be obtained from standard maximum likelihood procedures on the observed transformed durations implied by the first step estimates.

If a consistent but inefficient estimator $\hat{\theta}_{n}(W)$ of $\theta_{0}$ is available, e.g. the first stage estimator, and we have estimated the parameters of the polynomial approximation of the distribution of $U_{0}$ we can obtain an efficient estimator $\hat{\theta}_{\text {opt }}$ in just one additional step. From the linearization of the estimating equations, given in (C.3), we obtain an efficient estimator from

$$
\hat{\theta}_{\mathrm{opt}}=\hat{\theta}_{n}(W)-\hat{Q}(W)^{-1} S_{n}\left(\hat{\theta}_{n}(W) ; W_{\mathrm{opt}}\right) / n
$$

This procedure is related to obtaining an efficient GMM estimator in two steps from a consistent, but possible, inefficient GMM estimator. It also possible to obtain the efficient estimator directly from minimizing the quadratic form. However, this involves again the minimization of a multidimensional step function. 


\section{Application to the Illinois Re-employment Bonus Ex- periment}

Between mid-1984 and mid-1985, the Illinois Department of Employment Security conducted a controlled social experiment. ${ }^{6}$ This experiment provides the opportunity to explore, within a controlled experimental setting, whether bonuses paid to Unemployment Insurance (UI) beneficiaries or their employers reduce the time spend in unemployment relative to a randomly selected control group. In the experiment, newly unemployed claimants were randomly divided into three groups: a Claimant Bonus Group, a Employer Bonus Group and, a control group. The members of both bonus groups were instructed that they (Claimant group) or their employer (Employer group) would qualify for a cash bonus of $\$ 500$ if they found a job (of at least 30 hours) within 11 weeks and, if they held that job for at least four months. Each newly unemployed individual who was randomly assigned to one of the two bonus groups had the possibility to refuse participation in the experiment.

Woodbury and Spiegelman (1987) concluded from a direct comparison of the control group and the two bonus groups that the claimant bonus group had a significantly smaller average unemployment duration. The average unemployment duration was also smaller for the employer bonus group, but the difference was not significantly different from zero. These results are confirmed in Table 1. Note that the response variable is insured weeks of unemployment. Because UI benefits end after 26 weeks, all unemployment durations are censored at 26 weeks. In Table 1 no allowance is made for censoring. In the table we distinguish between compliers, those who agreed to be eligible for a bonus if assigned to a bonus group, and non-compliers. We see that the claimant bonus only affects the compliers and that the average unemployment duration of the non-compliers and the control group are almost equal.

Table 1: Average unemployment durations:control group and (non-)compliers.

\begin{tabular}{l|c|ccc|ccc}
\hline \hline & Control & \multicolumn{3}{|c|}{ Claimant } & \multicolumn{3}{c}{ Employer } \\
& Group & \multicolumn{3}{c|}{$\begin{array}{c}\text { Bonus } \\
\text { Bonus }\end{array}$} \\
& & All & Compl. & Non-compl. & All & Compl. & Non-compl. \\
\hline Benefit & 18.33 & 16.96 & 16.74 & 18.18 & 17.65 & 17.62 & 17.72 \\
weeks & & & & & & & \\
& $(0.20)$ & $(0.20)$ & $(0.22)$ & $(0.50)$ & $(0.21)$ & $(0.26)$ & $(0.35)$ \\
$\mathrm{N}$ & 3952 & 4186 & 3527 & 659 & 3963 & 2586 & 1377 \\
\hline \hline
\end{tabular}

standard error of average in brackets.

\footnotetext{
${ }^{6}$ A complete description of the experiment and a summary of its results can be found in Woodbury and Spiegelman (1987).
} 
About $15 \%$ of Claimant group and $35 \%$ of the employer group declined participation. The reason for this refusal is unknown. Bijwaard and Ridder (2005) showed that the participation rate is significantly related to some observed characteristics of the individuals that also influence that re-employment hazard. Hence, we cannot exclude the possibility of unmeasured variables that affect both the compliance decision and the re-employment hazard. Meyer (1996) analyzed the same data using a PH model with a piecewise constant baseline hazard. He used the randomization indicator instead of the actual bonus-group agreement indicator as an explanatory variable. Thus he used the ITT estimator. He found a significantly positive effect of the claimant bonus. However, as shown by Bijwaard and Ridder (2005), the ITT may have a downward bias.

We calculate the IVLR estimate of the effect of the claimant and employer bonus on the unemployment duration in a GAFT model and compare these estimates with the IVLR estimates of an AFT model, with ITT estimates in an MPH model and the ML estimates of an MPH model that ignores the endogeneity of the decision to participate in the bonus group. We consider the two interventions separately: thus Claimant Bonus group versus Control group and Employer Bonus group versus Control.

We shall consider two alternative specifications for the effect of the bonus on unemployment duration: $(i)$ constant effect and, $(i i)$ a change in the effect after 10 weeks, in line with the end of the eligibility period of the bonuses. Thus, the implied transformed durations are

$$
U(\theta)=\int_{0}^{T} \lambda(s ; \alpha) e^{\beta^{\prime} X+\left(\gamma_{1} I_{1}(s)+\gamma_{2} I_{2}(s)\right) D} \mathrm{~d} s
$$

with $I_{1}(t)=I(0 \leq t<11)$ and $I_{2}(t)$ is its complement. Note that the covariates are all timeconstant because the individual characteristics available in the data are all determined when the individuals register at the unemployment office. We include the following: the logarithm of the age (LNAGE), the logarithm of the pre-unemployment earnings (LNBPE), gender (MALE=1), ethnicity $(\mathrm{BLACK}=1)$, and the logarithm of the weekly amount of UI benefits plus dependence allowance (LNBEN). We employ two different specifications for $\lambda\left(t ; \alpha_{0}\right)$ : $(i)$ AFT model, i.e. $\lambda\left(t ; \alpha_{0}\right) \equiv 1$; and $(i i)$ GAFT model with a piecewise constant $\lambda$ on six intervals $0-2,2-4,4-6$, 6-10, 10-25 and 25 and beyond.

For identification we need to set one of the parameters of the piecewise constant $\lambda$ equal to one (or the log equal to zero). We let the base interval, the interval on which $\lambda=1$, start on the last week before the end of the observation period, at 25 weeks. This allows us to capture 
the spike in the observed unemployment duration just before the UI eligibility period ends. The end of the UI eligibility period, at 26 weeks, is for all individuals the same and thus provides the potential censoring time.

For both the AFT and the GAFT specifications we estimate a first stage IVLR using the Powell-method and the one step optimal IVLR. The first stage IVLR uses the values of the covariates, $X$, the interval indicators on the transformed duration (only for the GAFTmodel), $I_{j}(u)$ and, the bonus group assignment indicator times the interval indicators on the transformed duration, $R \cdot I_{1}(u)$ and $R \cdot I_{2}(u)$, as the weight functions. From these first stage IVLR's the implied transformed duration are obtained. Then, we estimate the parameters of the polynomial approximation of the distribution of $U$ conditional on $R$ and $D$ as mentioned in section 3.3. From these estimated parameters we calculate the hazard and its derivative of the transformed duration. These functions are then used as inputs to derive the optimal weight functions (see Theorem 3), which in turn are necessary to calculate the covariance matrix. We also calculate the 1-step efficient estimates with these optimal weight functions. In the case of a constant bonus effect, the optimal weight function are given in (15)-(17). When we assume that the effect of the bonus changes after 11 weeks the optimal weight function in (17) is more complicated and therefore not spelled out here.

The estimation results for the bonus effects are reported in Table 2. The results for the piecewise constant $\lambda$ and for the regression coefficients in the AFT and GAFT models can be found in appendix D. A comparison of the results shows that AFT overestimates the effect and that both ML and ITT estimators underestimate the effect of the employer bonus. For the claimant bonus the ML and ITT estimates are very close to the IVLR estimates. This indicates that endogeneity of the compliance decision is rather limited for the claimant group. The compliance rate in the claimant group is much higher and most probably the compliance decision of the individuals in the claimant bonus group is less related to their expected unemployment duration. The results clearly indicate that the bonuses only influence the chances to find a job in the first ten weeks. This is in line with the bonus eligibility period: those who find a job after that period would not get the bonus. The effect of the Claimant Bonus increases from about $10 \%$ higher probability to find a job at every unemployment duration to about $15 \%$ higher probability to find a job in the first ten weeks (and no effect thereafter). The bonus for the Employer group raises the job finding probability with about $7 \%$ at every unemployment duration or with about $12 \%$ in the first ten weeks of unemployment. 
Table 2: Instrumental Variable Linear Rank estimates for the effect of the Bonus

\begin{tabular}{|c|c|c|c|c|}
\hline \multicolumn{5}{|c|}{ Claimant group } \\
\hline Constant effect & AFT & GAFTа & MIF & ITT \\
\hline \multirow[t]{2}{*}{ First stage } & 0.1446 & 0.1024 & - & - \\
\hline & $(0.0493)$ & $(0.0523)$ & - & - \\
\hline \multirow[t]{2}{*}{ 1-step optimal } & 0.1596 & 0.0932 & 0.1039 & 0.1117 \\
\hline & $(0.0460)$ & $(0.0380)$ & $(0.0285)$ & $(0.0303)$ \\
\hline \multicolumn{5}{|c|}{ Time varying effect } \\
\hline \multicolumn{5}{|c|}{ First stage } \\
\hline \multirow[t]{2}{*}{$0-10$} & 0.2955 & 0.1433 & - & - \\
\hline & $(0.0523)$ & $(0.0907)$ & - & - \\
\hline \multirow[t]{2}{*}{$10+$} & -0.0720 & 0.0063 & - & - \\
\hline & $(0.0608)$ & $(0.0886)$ & - & - \\
\hline \multicolumn{5}{|l|}{ 1-step optimal } \\
\hline \multirow[t]{2}{*}{$0-10$} & 0.3865 & 0.1439 & 0.1601 & 0.1516 \\
\hline & $(0.0486)$ & $(0.0578)$ & $(0.0361)$ & $(0.0378)$ \\
\hline \multirow[t]{2}{*}{$10+$} & -0.0437 & -0.0411 & - & - \\
\hline & $(0.0572)$ & $(0.0850)$ & - & - \\
\hline
\end{tabular}

\begin{tabular}{|c|c|c|c|c|}
\hline \multicolumn{5}{|c|}{ Employer group } \\
\hline Constant effect & $\mathrm{AFT}$ & GAFT $^{\mathrm{a}}$ & MLE & ITT \\
\hline \multirow[t]{2}{*}{ First stage } & 0.1011 & 0.0721 & - & - \\
\hline & $(0.0646)$ & $(0.0470)$ & - & - \\
\hline \multirow[t]{2}{*}{ 1-step optimal } & 0.1332 & 0.0696 & 0.0387 & 0.0516 \\
\hline & $(0.0612)$ & $(0.0425)$ & $(0.0318)$ & $(0.0307)$ \\
\hline \multicolumn{5}{|c|}{ Time varying effect } \\
\hline \multicolumn{5}{|c|}{ First stage } \\
\hline \multirow[t]{2}{*}{$0-10$} & 0.2304 & 0.1103 & - & - \\
\hline & $(0.0710)$ & $(0.0736)$ & - & - \\
\hline \multirow[t]{2}{*}{$10+$} & -0.0783 & -0.0048 & - & - \\
\hline & $(0.0836)$ & $(0.1253)$ & - & - \\
\hline \multicolumn{5}{|l|}{ 1-step optimal } \\
\hline \multirow[t]{2}{*}{$0-10$} & 0.6334 & 0.1279 & 0.0881 & 0.0800 \\
\hline & $(0.0674)$ & $(0.0521)$ & $(0.0402)$ & $(0.0384)$ \\
\hline \multirow[t]{2}{*}{$10+$} & 0.0330 & -0.0747 & - & - \\
\hline & $(0.0745)$ & $(0.0882)$ & - & - \\
\hline
\end{tabular}

${ }^{\mathrm{a}}$ GAFT piecewise constant intervals: 0-2, 2-4, 4-6, 6-10, 10-25, $25 \rightarrow$ Notes: Standard error in brackets.

In the GAFT (and AFT) model the effect of the bonus is defined in terms of the change in the quantiles, see (3). In an AFT model with a time-constant coefficient for the bonus this effect is constant and independent of the other covariates. In a GAFT model the $\lambda$ function influences 
this effect directly and indirectly as the other covariates determine the quantiles. Using the distribution of $U_{0}$, already calculated to estimate the optimal IVLR and the variance-covariance matrix, we can derive the effect of the bonus in the GAFT depending on the quantile of the distribution. In Table 3 we present the effect of the bonus on the unemployment duration at the $80 \%, 60 \%$ and $40 \%$ survival for the reference individual and for a black individual, together with the AFT effect (first stage). Figure 1 till Figure 4 depict the change over the whole 90\%-25\% survival range of the effect of the bonus in the GAFT model.

Table 3: Effect of the Bonus on the length of unemployment duration

\begin{tabular}{|c|c|c|c|c|c|}
\hline \multirow{4}{*}{$\mathrm{AFT}$} & & \multicolumn{2}{|c|}{ Claimant } & \multicolumn{2}{|c|}{ Employer } \\
\hline & & Constant & Time-varying & Constant & Time-varying \\
\hline & $0-10$ & 0.865 & 0.744 & 0.904 & 0.794 \\
\hline & $10+$ & 0.865 & 1.075 & 0.904 & 1.081 \\
\hline GAFT & \multicolumn{5}{|c|}{ reference individual } \\
\hline \multirow[t]{3}{*}{$80 \%$} & $t_{q}(0)$ & 3.9 & 3.7 & 2.8 & 4.3 \\
\hline & $t_{q}(1)$ & 3.5 & 2.9 & 2.5 & 3.7 \\
\hline & effect & 0.911 & 0.866 & 0.933 & 0.823 \\
\hline \multirow[t]{3}{*}{$60 \%$} & $t_{q}(0)$ & 12.8 & 12.6 & 8.9 & 12.7 \\
\hline & $t_{q}(1)$ & 10.4 & 9.4 & 7.8 & 10.0 \\
\hline & effect & 0.911 & 0.571 & 0.933 & 1.078 \\
\hline \multirow[t]{3}{*}{$40 \%$} & $t_{q}(0)$ & 25.7 & 25.7 & 20.7 & 24.3 \\
\hline & $t_{q}(1)$ & 22.8 & 23.1 & 18.3 & 22.5 \\
\hline & effect & 1.772 & 1.973 & 0.933 & 1.078 \\
\hline GAFT & \multicolumn{5}{|c|}{ black individual } \\
\hline \multirow[t]{3}{*}{$80 \%$} & $t_{q}(0)$ & 7.5 & 6.8 & 4.8 & 8.1 \\
\hline & $t_{q}(1)$ & 6.5 & 5.3 & 4.1 & 6.4 \\
\hline & effect & 0.911 & 0.681 & 0.933 & 0.880 \\
\hline \multirow[t]{3}{*}{$60 \%$} & $t_{q}(0)$ & 25.3 & 24.4 & 18.44 & 24.22 \\
\hline & $t_{q}(1)$ & 22.1 & 21.0 & 16.2 & 22.5 \\
\hline & effect & 1.772 & 1.042 & 0.933 & 1.078 \\
\hline \multirow[t]{3}{*}{$40 \%$} & $t_{q}(0)$ & 35.6 & 35.1 & 30.7 & 34.2 \\
\hline & $t_{q}(1)$ & 32.9 & 33.8 & 28.90 & 33.9 \\
\hline & effect & 0.911 & 1.042 & 0.933 & 1.078 \\
\hline
\end{tabular}




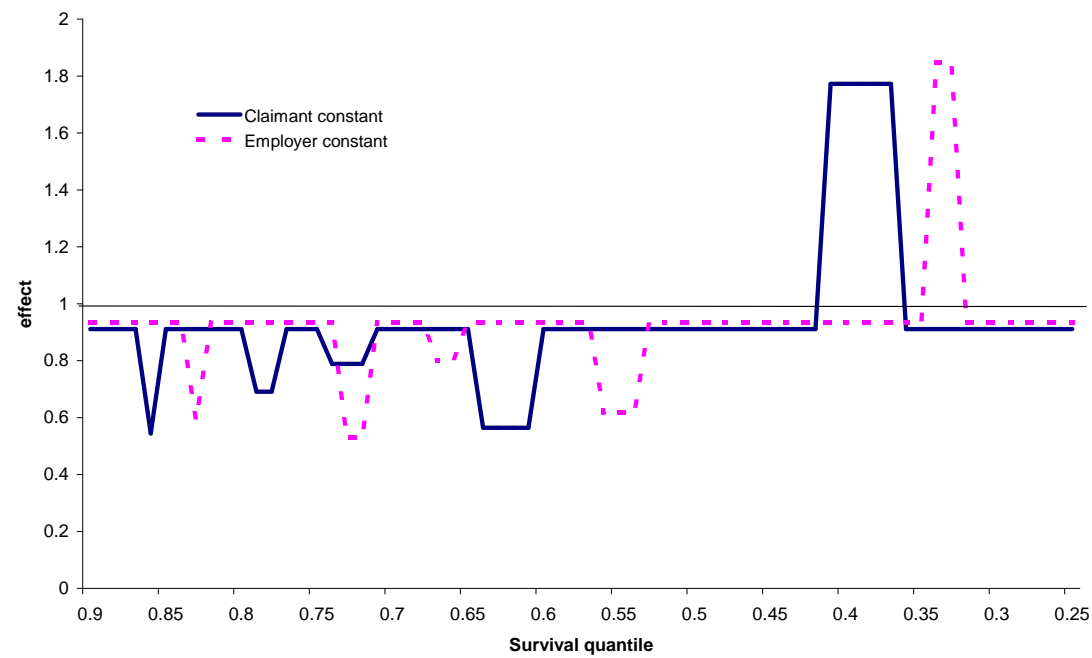

Figure 1: Effect of Bonus on quantiles of unemployment duration, $\frac{\mathrm{d} t_{q}(1)}{\mathrm{d} t_{q}(0)}$ (constant $\gamma$ )

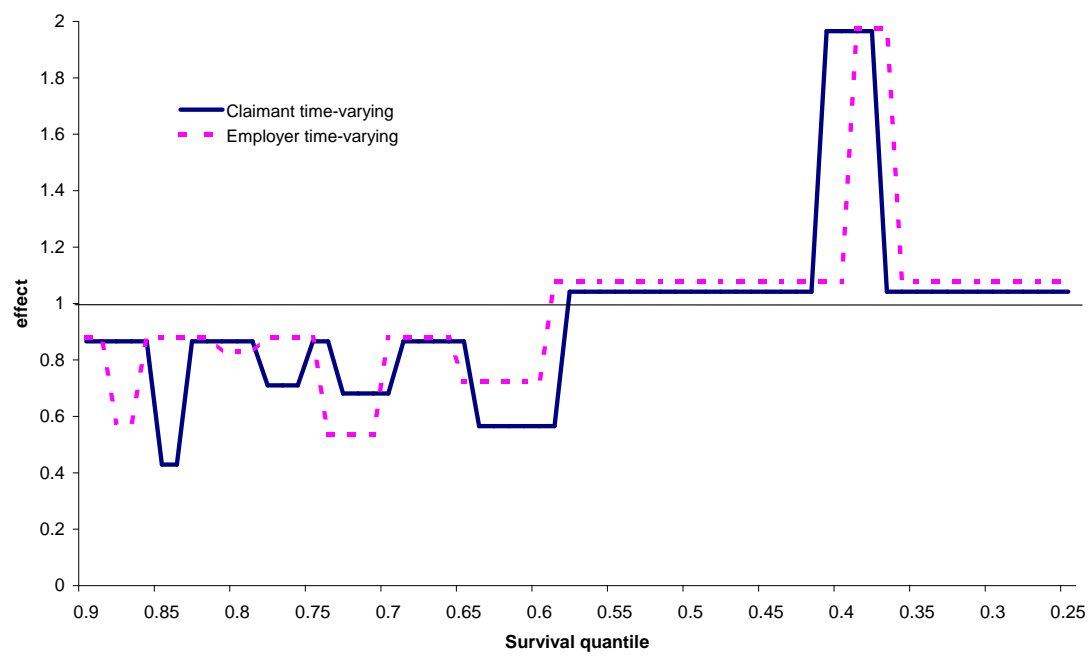

Figure 2: Effect of Bonus on quantiles of unemployment duration, $\frac{\mathrm{d} t_{q}(1)}{\mathrm{d} t_{q}(0)}$ (time-varying $\gamma$ )

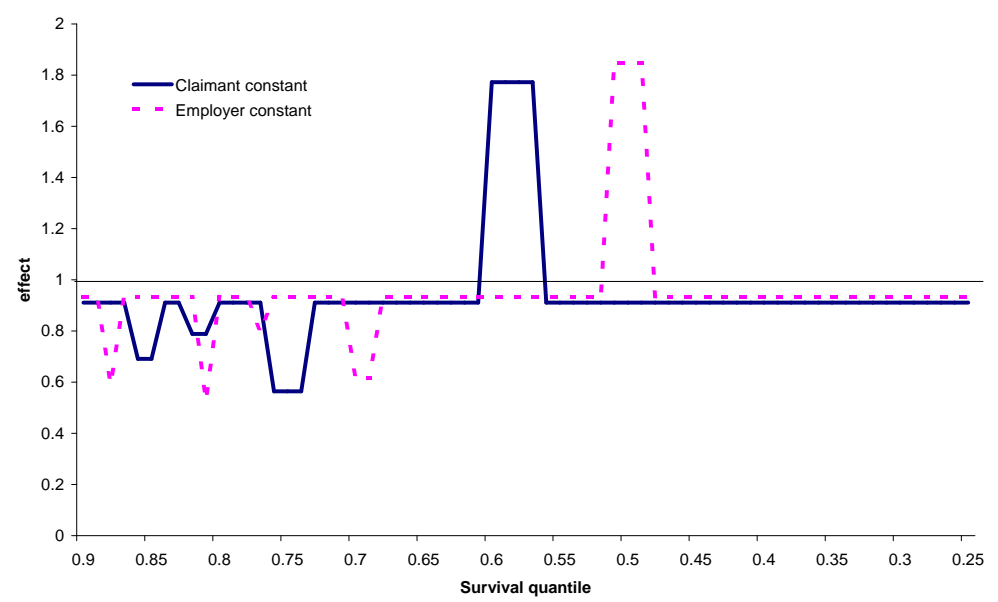

Figure 3: Effect of Bonus on quantiles of unemployment duration of BLACKS, $\frac{\mathrm{d} t_{q}(1)}{\mathrm{d} t_{q}(0)}$ (constant $\gamma)$ 


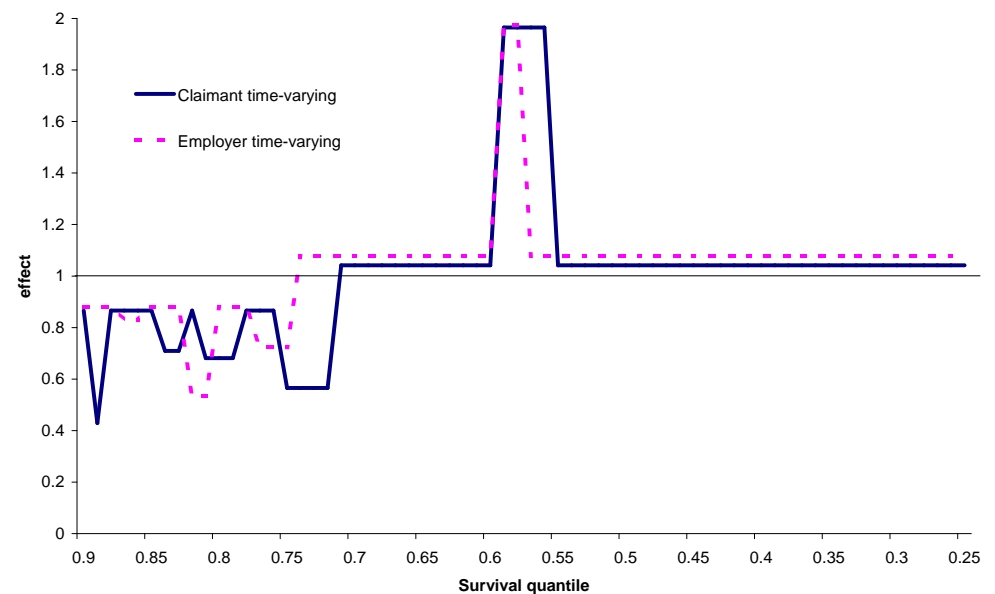

Figure 4: Effect of Bonus on quantiles of unemployment duration of BLACKS, $\frac{\mathrm{d} t_{q}(1)}{\mathrm{d} t_{q}(0)}$ (timevarying $\gamma$ ) 
Note that an effect smaller than one indicates that the bonus decreases the duration till re-employment and an effect bigger than one increases the duration. We see from the table (and more pronounced in Figure 1 and Figure 3) that even for a time-constant $\gamma$ the effect of the bonus on the unemployment duration in the GAFT model changes with the duration. The huge spike in the effect at the survival quantile of $40 \%$ for the claimant group is because the re-employment rate exhibits a spike just before the time that unemployment benefits are exhausted, which is at 26 weeks. For the individuals in the control group the $40 \%$ survival time is just before 26 weeks, while in the claimant bonus group it is at 23 weeks. Thus the control group individuals are in the re-employment spike while the claimant bonus group are not. The interval boundaries of the other intervals of $\lambda$ also cause, although not as pronounced, spikes. These spikes are downward because the $\lambda$ is jumping to a lower level at these boundaries. The spikes are also visible in the effect of a time-varying coefficient of the bonus, see Figure 2 and Figure 4. Here, the change in $\gamma$ at a duration of 10 weeks, after which the coefficient is negative, is reflected is a upward shift of the effect curve.

An indication that the AFT is not the right model is the difference between the first stage and one-step optimal estimators for the AFT model. For a correctly specified model both estimators are consistent and, therefore, do not differ much. In the GAFT model the first stage and one-step estimator are of the same magnitude. The estimated standard errors of the latter are, as expected, substantially lower in most situations.

Although the focus in this article is on the estimation of the effect of a possibly endogenous variable on the duration we also give a short discussion on the estimation results of the other parameters. These estimators can be found in the tables in appendix D. The regression parameters are overestimated (in absolute terms) if we assume an AFT model. These regression parameters hardly change from a model with constant bonus effect (Table 5) to a model with time-varying bonus effect (Table 6). The regression parameters for the Claimant data and the Employer data (both including the control group) are almost identical. Gender, MALE, is the exception; Gender has no significant influence on the re-employment probability in the Employer data. The shape of the estimated $\lambda$ 's indicate a U-shaped $\lambda$.

We end with a discussion on the selectivity in the bonus data. The compliance rate in the Claimant group, $85 \%$, was much higher than the compliance rate in the employer group, $65 \%$. Many individuals in the Employer group, apparently and contrary to our findings, did not perceive a bonus paid to their new employer beneficiary for their job search. Following Moffitt 
(1983) this partial compliance may be explained by a stigma effect. However, this is a tentative explanation because our analysis only adjust for (possible) selective compliance. It does not provide a model for the selection process. Thus, both an advantage and a drawback of our method is that we do not make any assumptions on the selection process and therefore cannot tell why individuals make such a selective decision.

\section{Conclusion}

In this article we proposed and implemented an instrumental variable estimation procedure for duration models. We show how the effect of an endogenous variable on the duration in a Generalized Accelerated Failure Time (GAFT) model can be estimated. The GAFT model is based on a transformation of the durations that encompasses both the Accelerated Failure Time (AFT), very popular in biostatistics, and the Mixed Proportional Hazards (MPH) model, very popular in econometrics. The interpretation of regression coefficients in the GAFT is in terms of shifting the quantiles of the distribution.

The basis of the Instrumental Variable Linear Rank estimator is that for the true GAFT model the instrument does not influence the hazard of the transformed duration. This implies that a rank test on the significance of the effect of instrument on the hazard of the transformed duration is zero. The IVLR estimation procedure is based on the inverse of an extended, including all the parameters of the GAFT model, rank-test. The estimation procedure is related to the rank estimation procedures of Robins and Tsiatis (1991) and of Bijwaard and Ridder (2005). The Two Stage Linear Rank procedure of Bijwaard and Ridder (2005) is based on a semi-parametric MPH and requires preliminary estimates of the baseline hazard. The Rank Preserving Structural Failure Time Model of Robins and Tsiatis (1991) is based on the strong version of the Accelerated Failure Time model. Their model imposes a strong non-interaction assumption. This implies that if two individuals have the identical observed durations and observed treatment histories then they would have had identical durations had treatment always been withheld. The IVLR estimator does neither impose the non-interaction assumption nor requires preliminary estimates of the baseline hazard.

The estimation procedure is also related to quantile-regression, in particular Koenker and Bilias (2001) and Koenker and Geling (2001). It is, however, unclear how these methods can handle time-varying endogenous variables. Because the IVLR is based on a vector of mean restrictions it is related to the well-known GMM estimation procedure. Similar to the application 
of GMM estimation choosing the right weight functions can improve the efficiency. However, again similar to the GMM, these optimal weight functions are not directly observable. Fortunately, an adaptive (or even 2 step) procedure can provide the efficient IVLR.

We can give a causal interpretation to the effect of the endogenous variable the IVLR identifies for the GAFT model. However, the causal effect is defined in terms of shifting the quantiles of the outcome distribution and not in terms of the (Local) Average Treatment Effect, common in the treatment evaluation literature. But averages are less usefull to base treatment effects on for duration data, due to censoring and time-varying treatment.

The empirical application shows that the ML and ITT estimates for the employer group, in which the new employer of the claimant receives the bonus, are downward biased due to endogeneity. In the claimant group, in which the claimant himself receives the bonus, the ML and ITT estimates are close to the IVLR estimates. This might indicate that the endogeneity of the decision to participate in this group is rather small. Incorrectly assuming an AFT model can give misleading conclusions about the effects of a bonus on the re-employment hazard. In the Illinois bonus re-employment experiment many unemployed found a job just before their UI-benefits expires. This induces a spike in the re-employment hazard. In the GAFT, even with a constant regression coefficient, such a spike leads to an effect that changes over the quantiles. This has important implications for the evaluation of the effect of a possible endogenous variable on a duration.

Social experiments may provide instruments for an endogenous variable. With good instruments available the proposed method can be very useful in analyzing the effects of a possible endogenous variable on an inherently duration outcome. Examples in population studies include the effect of training programs on the unemployment duration, policies to increase the birth rate and migration policies.

There are several issues that need further research. First, the current approach to adjust for endogenous censoring implies loss of information and depends on the (unknown) parameters of the model. An important improvement would be to find a method to adjust for endogenous censoring that is parameter independent and minimizes the loss of information. Another related issue is that if the IVLR assumes that the censoring time is (potentially) known in advance. Further research on more general censoring patterns deserve attention. Second, in our empirical application we have, because of random assignment, a perfect assignment. Such an instrument is, however, not always available. Finding good instruments is therefore an important issue just 
as the influence of weak instruments on the properties of the estimator. A final issue for further research is the extension of the IVLR to recurrent duration data, like repeated unemployment spells.

\section{References}

Abbring, J. H. and G. J. van den Berg (2005). Social experiments and instrumental variables with duration outcomes. Tinbergen Institute, discussion paper, TI 2005-47.

Andersen, P. K., O. Borgan, R. D. Gill, and N. Keiding (1993). Statistical Models Based on Counting Processes. New York: Springer-Verlag.

Angrist, J. D. and A. B. Krueger (1999). Empirical strategies in labor economics. In O. Ashenfelter and D. Card (Eds.), Handbook of Labor Economics, Volume 3A, Chapter 23, pp. 1277-1366. Amsterdam: North-Holland.

Ashenfelter, O., D. Ashmore, and O. Deschêne (2005). Do unemployment insurance recipients actively seek work? Evidence from randomized trials in four U.S. states. Journal of Econometrics 125, 53-75.

Bijwaard, G. E. and G. Ridder (2005). Correcting for selective compliance in a re-employment bonus experiment. Journal of Econometrics 125, 77-111.

Brännäs, K. (1992). Econometrics of the Accelerated Duration Model. Umeå: Solfjädern Offset AB.

Cox, D. R. and D. Oakes (1984). Analysis of Survival Data. London: Chapman and Hall.

Han, A. K. (1987). Non-parametric analysis of a generalized regression model: The maximum rank correlation estimator. Journal of Econometrics 35, 303-316.

Heckman, J. J., R. J. LaLonde, and J. A. Smith (1999). The economics and econometrics of active labor market programs. In O. Ashenfelter and D. Card (Eds.), Handbook of Labor Economics, Volume 3A, Chapter 31, pp. 1866-2097. Amsterdam: North-Holland.

Kalbfleisch, J. D. and R. L. Prentice (2002). The Statistical Analysis of Failure Time Data (second edition). John Wiley and Sons.

Klein, J. P. and M. L. Moeschberger (1997). Survival Analysis: Techniques for Censored and Truncated Data. New York: Springer-Verlag. 
Koenker, R. and Y. Bilias (2001). Quantile regression for duration data: A reappraisal of the Pennsylvania reemployment bonus experiments. Empirical Economics 26, 199-220.

Koenker, R. and O. Geling (2001). Reappraising medfly longevity: A quantile regression survival analysis. Journal of the American Statistical Association 96, 458-468.

Lalive, R., J. C. van Ours, and J. Zweimüller (2005). The effect of benefit sanctions on the duration of unemployment. Journal of the European Economic Association 3, 1386-1417.

Meyer, B. D. (1995). Lessons from the U.S. unemeployment insurance experiments. Journal of Economic Literature 33, 91-131.

Meyer, B. D. (1996). What have we learned from the Illinois reemployment bonus experiment? Journal of Labor Economics 14, 26-51.

Moffitt, R. (1983). An economic model of welfare stigma. American Economic Review 73, 1023-1035.

Powell, M. J. D. (1964). An efficient method for finding the minimum of a function of several variables without calculating derivatives. The Computer Journal 7, 155-162.

Prentice, R. L. (1978). Linear rank tests with right censored data. Biometrika 65, 167-179.

Press, W. H., B. P. Flannert, S. A. Teukolsky, and W. T. Vetterling (1986). Numerical Recipes: The Art of Scientific Computing. Cambridge: Cambridge UP.

Ridder, G. (1990). The non-parametric identification of generalized accelerated failure-time models. Review of Economic Studies 5\%, 167-182.

Robins, J. M. and A. A. Tsiatis (1991). Correcting for non-compliance in randomized trials using rank-preserving structural failure time models. Communications in Statistics Part A: Theory and Methods 20, 2609-2631.

Tsiatis, A. A. (1990). Estimating regression parameters using linear rank tests for censored data. Annals of Statistics 18, 354-372.

Van den Berg, G. J. (2001). Duration models: Specification, identification, and multiple duration. In J. Heckman and E. Leamer (Eds.), Handbook of Econometrics, Volume V, Chapter 55, pp. 3381-3460. Amsterdam: North-Holland.

Van den Berg, G. J., J. C. van Ours, and B. van der Klaauw (2004). Punitive sanctions and the transition rate from welfare to work. Journal of Labor Economics 22, 211-241. 
Woodbury, S. A. and R. G. Spiegelman (1987). Bonuses to workers and employers to reduce unemployment: Randomized trials in Illinois. American Economic Review 7\%, 513-530.

Ying, Z. (1993). A large sample study of rank estimation for censored regression data. Annals of Statistics 21, 76-99. 


\section{A Identification of the GAFT model}

Assume that the regression function in the GAFT model is log-linear. Then, the model is characterized by the non-negative function $\lambda(t ; \alpha)$ defined on $[0, \infty)$, the distribution of $U_{0}$ and the regression parameter $\beta$. Ridder (1990) has shown that if the covariates are time constant, all observationally equivalent GAFT models, i.e. models that give the same conditional distribution of $T$ given $X$, have regression parameters $d \beta$, integrated transformation $c_{1}\left(\int_{0}^{t} \lambda_{0}\left(s ; \alpha_{0}\right) d s\right)^{c_{2}}$ and $U_{0}$ distribution $G_{0}\left(\left(\frac{u}{c_{1}}\right)^{1 / c_{2}}\right)$ for some constants $c_{1}, c_{2}>0$. The equivalent class follows from the fact that a GAFT model with time constant covariates can be expressed as a transformation model

$$
\ln \left(\int_{0}^{T} \lambda_{0}\left(s ; \alpha_{0}\right) \mathrm{d} s\right) \stackrel{d}{=}-\beta_{0}^{\prime} X+\ln U
$$

and the constants $c_{1}, c_{2}$ correspond to addition of $e^{c_{1}}$ to and division by $c_{2}$ of the left- and right-hand sides.

With time-varying covariates, the set of observationally equivalent GAFT models is generally smaller. In particular, the power transformation that gives an observationally equivalent model if the covariates are time constant, in general does not result in a GAFT model. As an example consider the GAFT model with time-varying regressors that differ between two groups. In group $I$

$$
X(t)= \begin{cases}1 & \text { if } 0 \leq t \leq 1 \\ 0 & \text { if } t>0\end{cases}
$$

and in group $I I, X(t)=0 ; t \geq 0$. Moreover $\lambda_{0}(t ; \alpha)=\alpha t^{\alpha-1}$. With time constant regressors the parameter $\alpha$ is not identified. It can be shown that the observationally equivalent GAFT models have transformation $c_{1} t^{\alpha}$ and $U$-distribution with survival $G_{u}\left(\frac{u}{c_{1}}\right)$. Hence, with time-varying covariates $\alpha$ is identified (and so is $\beta$ ).

We conclude that identification depends on whether the covariates are time constant or timevarying. If the covariates are time constant we can identify the transformation $h\left(T, \bar{X}(T) ; \theta_{0}\right)$ up to a power and $\beta$ up to scale (with the power and the scale being equal). Moreover, if we fix the power we can identify $h\left(T, \bar{X}(T) ; \theta_{0}\right)^{c_{2}}$ up to scale and the distribution of $U_{0}$ up to the same scale parameter.

If the covariates are time-varying we can, except in special cases, identify $h\left(T, \bar{X}(T) ; \theta_{0}\right)$ and the distribution of $U_{0}$ up to a common scale parameter. Because we leave the distribution of $U_{0}$ unspecified in our estimation method, we can not use restrictions on $U_{0}$ to find the scale parameter. For that reason we normalize $h\left(T, \bar{X}(T) ; \theta_{0}\right)$ by setting $h\left(T, 0 ; \theta_{0}\right)=1$ for some $t_{0}>$ 
0. With time constant regressors we need the same normalisation, but in addition we need to set one regression coefficient equal to one. Of course, we could choose a class of transformations that is not closed under the power transformation. This amounts to identification by functional form.

Finally, we need a condition on the sample paths of $X$ in the population. If we rewrite (1) as

$$
\int_{0}^{T} e^{\ln \lambda\left(s ; \alpha_{0}\right)+\beta_{0}^{\prime} X(s)} \mathrm{d} s=U_{0}
$$

we require that

$$
\operatorname{Pr}\left(\ln \lambda\left(s ; \alpha_{0}\right)+\beta_{0}^{\prime} X(.)=0\right)=0
$$

where the probability is computed over the distribution of $X$ as a random function of $t$ and 0 is the zero function. In other words, $\ln \lambda$ is not collinear with $X$.

For the identification in the GAFT model with endogenous variables we need additional assumptions on the instrument. First, the instrument should only affect the duration through the endogenous variable and not directly. Second, the value of the instrument should influence the value of the endogenous variable in a non-trivial way. For example, if both the instrument and the endogenous variable are binary then $\operatorname{Pr}(D=1 \mid R=1)>0$ and $\operatorname{Pr}(D=0 \mid R=0)>0$.

\section{B Counting process interpretation}

The density and the survival function of a duration $T$ can be expressed as functions of the hazard rate. These expressions can be used to obtain a likelihood function. In this appendix we use a different (but of course equivalent) representation of the relation between the hazard rate and the random duration. In particular, we use the framework of counting processes (see e.g. Andersen et al. (1993) and Klein and Moeschberger (1997)). The main advantage of this framework is that it allows us to express the duration distribution as a regression model with an error term that is a martingale difference. This simplifies the analysis of the estimator. The conditions for non selective observation can be precisely stated in this framework. The same is true for conditions on time--varying covariates.

The starting point is that the hazard of $T$ is the intensity of the counting process $\{N(t) ; t \geq$ $0\}$ that counts the number of times that the event occurs during $[0, t]$. The counting process has a jump +1 at the time of occurrence of the event ${ }^{7}$. A jump occurs if and only if $\mathrm{d} N(t)=$

\footnotetext{
${ }^{7}$ The sample paths are assumed to be right-continuous.
} 
$N(t)-N(t-)=1$. For duration data, the event can only occur once. In many unemployment studies the individuals are only observed until re-employment. So, at most one jump is observed for any unit. To account for this we introduce the observation indicator $Y(t)=I(T \geq t)$ that is zero after re-employment. By specifying the intensity as the product of this observation indicator and the hazard rate we effectively limit the number of occurrences of the event to one. We assume that the observation indicator only depends on events up to time $t$. The observation process is assumed to have left-continuous sample paths. We define the history of the process up to time $t$ by $H(t)=\{\bar{Y}(t), D, \bar{X}(t)\}$, where $\bar{Y}(t)=\{Y(s), 0 \leq s \leq t\}$. The history $H(t)$ only contains observable events.

Let $V$ be some unobserved variables that both influence the endogenous variable and the duration. An example is the, usually, unobserved search intensity of unemployed looking for a job. We assume that $V$ and $\bar{X}(t)$ are stochastically independent. Denote $H^{V}(t)=\{H(t), V\}$, the history that also includes the unobservables. As with dynamic regressors in time-series models, the time-varying $X(t)$ may depend on the dependent variable up to time $t$ but not after time $t$ (conditionally on $V$ ). Thus $D$ only depends on $H^{V}(t)$ and $X(t)$ only on $H(t)$. In the counting process literature such a time-varying covariate is called predictable. We will use the econometric term predetermined.

If the conditional distributions of $N(t)$ given $H^{V}(t)$ or $H(t)$ are well-defined (see Andersen et al. (1993) for assumptions that ensure this) we can express the probability of an event in $(t-d t, t] \mathrm{as}^{8}$

$$
\operatorname{Pr}\left(\mathrm{d} N(t)=1 \mid H^{V}(t)\right)=Y(t) \kappa(t \mid \bar{X}(t), D, V) \mathrm{d} t
$$

with $\kappa(t \mid \cdot)$ is the hazard of $T$ at $t$ given $\bar{X}(t), D$ and $V$. By the Doob-Meier decomposition

$$
\mathrm{d} N(t)=Y(t) \kappa(t \mid \bar{X}(t), D, V) \mathrm{d} t+\mathrm{d} M(t)
$$

with $\{M(t) ; t \geq 0\}$ a (local square integrable) martingale. The conditional mean and variance of this martingale are

$$
\begin{aligned}
\mathrm{E}(\mathrm{d} M(t) \mid H(t)) & =0 \\
\operatorname{Var}(\mathrm{d} M(t) \mid H(t)) & =Y(t) \kappa(t \mid \bar{X}(t), D, V) \mathrm{d} t
\end{aligned}
$$

The (conditional on $H(t))$ mean and variance of the counting process are equal, so that the

\footnotetext{
${ }^{8}$ Because the sample paths of $\{Y(t), X(t), t \geq 0\}$ are assumed to be left-continuous (as is the baseline hazard), we can substitute $t$ for $t-d t$ in (B.1).
} 
disturbances in equation (B.2) are heteroscedastic. The probability in equation (B.1) is zero, if the individual is not at risk.

A counting process can be considered as a sequence of Bernoulli experiments, because if $\mathrm{d} t$ is small equations (B.1) and (B.4) give the mean and variance of a Bernoulli random variable. The relation between the counting process and the sequence of Bernoulli experiments is given in equation (B.2), which can be considered as a regression model with an additive error that is a martingale difference. This equation resembles a time-series regression model. The Doob-Meier decomposition is the key to the derivation of the distribution of the estimator, because the asymptotic behavior of partial sums of martingales is well-known.

The GAFT model transforms the observed duration $T$ to a transformed duration $U_{0}$. The transformation involved a parameter vector $\theta_{0}=\left(\beta_{0}^{\prime}, \gamma_{0}^{\prime}, \alpha_{0}^{\prime}\right)^{\prime}$. We denote the transformation for parameter vectors $\theta \neq \theta_{0}$ by $U(\theta)$ with $U_{0}=U\left(\theta_{0}\right)$. The distribution of $U(\theta)$ can also be represented by a (transformed) counting process $\left\{N^{U}(u) ; u \geq 0\right\}$. The relation between the original and transformed counting process, the observation indicator, and the time-varying exogenous covariates is

$$
\begin{array}{ll}
N^{U}(u ; \theta)=N\left(h^{-1}(u ; \theta)\right) & Y^{U}(u ; \theta)=Y\left(h^{-1}(u ; \theta)\right) \\
X^{U}(u ; \theta)=X\left(h^{-1}(u ; \theta)\right) & I_{k}^{U}(u ; \theta)=I_{k}\left(h^{-1}(u ; \theta)\right)
\end{array}
$$

with $h(T ; \theta)=h(T, \bar{X}(T), \bar{D}(T) ; \theta)$, defined in $(4)$, and $I_{k}(t)=I\left(t_{k}<t \leq t_{k+1}\right)$. For $\theta=\theta_{0}$ we denote $h_{0}(T)=h\left(T ; \theta_{0}\right)$. The corresponding history is $H^{U}(u ; \theta)=\left\{\bar{Y}^{U}(u ; \theta), \bar{X}^{U}(u ; \theta), \bar{I}_{k}^{U}(u ; \theta), D\right\}$. In the sequel we suppress $\theta$ and write $Y^{U}(u), N^{U}(u), \bar{X}^{U}(u), \bar{I}_{k}^{U}(u)$ and $H^{U}(u)$ for $\theta \neq \theta_{0}$ and $Y_{0}(u), N_{0}(u), \bar{X}_{0}(u), \bar{I}_{k 0}(u)$ and $H_{0}(u)$ for $\theta=\theta_{0}$. The intensity of the transformed counting process with respect to history $H^{U}(u)$ is obtained by the innovation theorem (see Andersen et al. (1993), p. 80, 87) ${ }^{9}$

$$
\begin{aligned}
\operatorname{Pr}\left(\mathrm{d} N^{U}(u)=1 \mid H^{U}(u)\right) & =Y^{U}(u) \mathrm{E}\left[\frac{\lambda\left(h^{-1}(u ; \theta) ; \alpha_{0}\right)}{\lambda\left(h^{-1}(u ; \theta) ; \alpha\right)} e^{\left(\beta_{0}-\beta\right)^{\prime} X^{U}(u)}\right. \\
& \left.\times \exp \left(\sum_{k=1}^{K}\left(\gamma_{k 0}-\gamma_{k}\right) I_{k}^{U}(u) D\right) \kappa_{0}\left(h_{0}\left(h^{-1}(u ; \theta)\right)\right) \mid H^{U}(u)\right] \mathrm{d} u
\end{aligned}
$$

\footnotetext{
${ }^{9}$ If $U=h(T)$ and $\kappa_{T}$ is the hazard rate of the distribution of $T$, then the hazard rate of the distribution of $U$ is

$$
\kappa_{U}(u)=\kappa_{T}\left(h^{-1}(u)\right) \frac{1}{h^{\prime}\left(h^{-1}(u)\right)}
$$
}


We implicitly integrate with respect to the distribution of the unobserved $V$ conditional on $H^{U}(u)$. Note that these unobserved covariates are only introduced to ascertain the predictability of the endogenous covariate process. Although the distribution of those variables determines the distribution of $U_{0}$, the consistency of the IVLR is independent of that distribution. Unfortunately, even for the population parameters $\theta_{0}$ the hazard of $U_{0}, \kappa_{0}(u)$, still depends on the intervention path (through the correlation with $V$ ). If we condition on the history of the instruments instead of the actual endogenous covariates we do get the desired independence.

We must add the instrument $R$ to the conditioning variables in (B.5) if we consider instrumenting the endogenous variable. Let the $U R$-history, $H^{U R}(u)=\left\{Y^{U}(s), X^{U}(s), R ; 0 \leq s \leq u\right\}$, be the history on the transformed durations in which the endogenous variable $D$ is replaced by the instrument. Then, another application of the innovation theorem gives the intensity of the transformed process on the $U R$-history

$$
\begin{aligned}
\operatorname{Pr}\left(\mathrm{d} N^{U}(u)=1 \mid H^{U R}(u)\right) & =Y^{U}(u) \mathrm{E}\left[\frac{\lambda\left(h^{-1}(u ; \theta) ; \alpha_{0}\right)}{\lambda\left(h^{-1}(u ; \theta) ; \alpha\right)} e^{\left(\beta_{0}-\beta\right)^{\prime} X^{U}(u)}\right. \\
\times & \left.\exp \left(\sum_{k=1}^{K}\left(\gamma_{k 0}-\gamma_{k}\right) I_{k}^{U}(u) D\right) \kappa_{0}\left(h_{0}\left(h^{-1}(u ; \theta)\right)\right) \mid H^{U R}(u)\right] \mathrm{d} u
\end{aligned}
$$

which for the population parameters simplifies to $Y_{0}^{U}(u) \kappa_{0}(u) \mathrm{d} u$ with $H_{0}^{U R}(u)=H^{U R}\left(u ; \theta_{0}\right)$. Note that (B.5) and (B.6) only differ in the history the intensities are conditioned on.

For further reference we denote the intensity in (B.6) by $\kappa_{i}^{U}(u ; \theta)$ such that

$$
\operatorname{Pr}\left(\mathrm{d} N^{U}(u)=1 \mid H^{U R}(u)\right)=Y^{U}(u) \kappa_{i}^{U}(u ; \theta) \mathrm{d} u
$$

which reduces to $\kappa_{0}(u)$ for the population parameters.

A common feature of duration data is that some of the observations are censored. Assume the censoring time, $C$, is (potentially) known. Then, the potential censoring time is known and the observed durations are $\tilde{T}=\min (T, C)$ and $\Delta=I(T \leq C)$, where $\Delta$ is one if $T$ is observed.

Assume the piecewise constant structure for the effect of the endogenous variable in (5). This implies that for $t_{k}<t \leq t_{k+1}$, the coefficient of $D=1$ is $e^{\gamma_{k}}$. We define the transformed censoring time $C^{U}(\theta)$ (possibly depending on the observed history of other covariates) such that: (a) $T \geq C$ implies $h(T ; \theta) \geq C^{U}(\theta)$ and (b) $U_{0}$ and $R$ are independent on the interval bounded above by $C^{U}(\theta)$.

Note that we either observe $T \leq C$ and $\Delta=1$, or $T>C$ and $\Delta=0$. If some of the other covariates are also time-varying we have another identification problem, because these 
covariates are only observed up until $\tilde{T}$. The transformed censoring times (conditional on $T, C>$ $t_{k}$ ) that take all these considerations into account are the sum of the transformed duration up to $t_{k}, h\left(t_{k} ; \theta\right)$ and the censoring adjustment, i.e

$$
C^{U}(\theta)= \begin{cases}\int_{0}^{C} \lambda(s ; \alpha) e^{\beta^{\prime} X(s)} P(s ; \gamma) \mathrm{d} s & \text { if } T>C \\ \int_{0}^{T} \lambda(s ; \alpha) e^{\beta^{\prime} X(s)} P(s ; \gamma) \mathrm{d} s+\int_{T}^{C} \lambda(s ; \alpha) d s & \text { if } T \leq C .\end{cases}
$$

where $P(s ; \gamma)=I\left(s \geq t_{k}\right) \prod_{j=0}^{k} \min \left(e^{\gamma_{j}}, 1\right)$. From the last term on the right-hand side of (B.7) we see why we need to know $C$ even for the uncensored observations. Otherwise we can not compute $C^{U}(\theta)$ for these observations. We can estimate the parameters of the Instrumental GAFT model from the following observed data

$$
\tilde{U}(\theta)=\min \left(U(\theta), C^{U}(\theta)\right), \quad \Delta^{U}(\theta)=I\left(U(\theta)<C^{U}(\theta)\right)
$$

and $Y^{U}(u ; \theta)=I(\tilde{U}(\theta) \geq u)$. Now $\tilde{U}\left(\theta_{0}\right)$ is independent of $R$ for $\Delta^{U}\left(\theta_{0}\right)=1$. Note that if, at least, one of the $\gamma$ 's is different from zero, we introduce extra censoring on the transformed durations, because then some units with $\Delta=1$ have $\Delta^{U}(\theta)=0$.

The counting process interpretation allows for an alternative formulation of the estimating equations in (8). The relevant counting measure, $N_{i}^{U}(u)$, can be seen as a discrete 'probability distribution' that assigns weight unity to uncensored transformed durations and is zero elsewhere. Then the estimating equations can be expressed as an integral with respect to that counting process

$$
S_{n}(\theta ; W)=\sum_{i=1}^{n} \int_{0}^{C_{i}^{U}}\left\{W\left(u, R_{i}\right)-\bar{W}(u ; \theta)\right\} \mathrm{d} N_{i}^{\tilde{U}}(u)
$$

where $C_{i}^{U}$ is the transformed censoring time defined in (B.7).

\section{Asymptotic properties of the IVLR}

In this section we discuss the asymptotic behavior of the Instrumental Variable Linear Rank estimator. The counting process framework simplifies the derivation of these asymptotic properties. We assume a piecewise constant $\lambda$ for the GAFT model.

We make the following assumptions:

C1: The covariate process $X(t)$ is predetermined, i.e. its distribution is independent of $\{H(s), s>$ $t\}$. The sample paths of the covariate process are bounded and at least one of time-varying covariates is a continuous variable. 
C2: The observation process $Y(t)$ is cadlag and $Y(t)$ is predetermined. Moreover,

$$
\operatorname{Pr}(\mathrm{d} N(t)=1 \mid Y(t)=1, H(t))=\operatorname{Pr}(\mathrm{d} N(t)=0 \mid Y(t)=0, H(t))
$$

C3: The population distribution of $T$ given $\bar{X}$ and $\bar{D}$ satisfies

$$
\int_{0}^{T} \lambda\left(s ; \alpha_{0}\right) e^{\beta_{0}^{\prime} X(s)+\psi\left(s, D ; \gamma_{0}\right)} \mathrm{d} s=U_{0}
$$

The absolutely continuous distribution of $U_{0}$ does not depend on $\bar{X}$ or $\bar{R}$. The p.d.f. of $U_{0}$ is bounded.

C4: The transformed observation process $Y^{U}(u)=I(\tilde{U}(\theta) \geq u)$ is cadlag and predetermined, with $\tilde{U}(\theta)=\min \left(U(\theta), C^{U}\right)$ and $C^{U}$ defined in (B.7).

C5: The instrumental function $W$ is bounded and left-continuous.

C6: The intensity of $U(\theta), \kappa_{i}^{U}(u)$ given history $H^{U R}(u)$ in (B.6) can be linearized in a neighborhood of $\theta_{0}$ as a function of $\theta$, i.e. there exist $\mu(u)$ and $\epsilon>0$ such that for $\left\|\theta-\theta_{0}\right\|<\epsilon$

$$
\left|\kappa_{i}^{U}(u ; \theta)-\kappa_{0}(u)-\left(\theta-\theta_{0}\right)^{\prime} d_{i 0}(u)\right| \leq\left\|\theta-\theta_{0}\right\|^{2} \mu(u)
$$

for $u \leq C_{0}=C^{U}\left(\theta_{0}\right)$ with

$$
d_{i 0}(u)=\left.\frac{\partial \kappa_{i}^{U}(u ; \theta)}{\partial \theta}\right|_{\theta=\theta_{0}}
$$

C7: There exists a continuous function $a(u ; \theta)$ of $\theta$ in a neighborhood $B$ of $\theta_{0}$ such that

$$
\sup _{u \leq C_{0}} \sup _{\theta \in B}\|\bar{W}(u ; \theta)-a(u ; \theta)\| \stackrel{p}{\rightarrow} 0
$$

where

$$
\bar{W}(u ; \theta)=\frac{\sum_{j=1}^{n} Y_{j}^{U}(u) W\left(u, \bar{X}_{j}^{U}(u), R_{j}\right)}{\sum_{j=1}^{n} Y_{j}^{U}(u)}
$$

C8: There exists a continuous matrix function $A(u ; \theta)$ of $\theta$ in a neighborhood $B$ of $\theta_{0}$ such that

$$
\begin{aligned}
\sup _{u \leq C_{0}} \sup _{\theta \in B} \| \frac{1}{n} \sum_{i=1}^{n}\left[W \left(u, \bar{X}_{i}^{U}(u),\right.\right. & \left.\left.R_{i}\right)-\bar{W}(u ; \theta)\right] \\
\times & {\left[W\left(u, \bar{X}_{i}^{U}(u), R_{i}\right)-\bar{W}(u ; \theta)\right]^{\prime} Y_{i}^{U}(u)-A(u ; \theta) \| \stackrel{p}{\rightarrow} 0 }
\end{aligned}
$$

C9: There exists a continuous matrix-function $V(u ; \theta)$ of $\theta$ in a neighborhood $B$ of $\theta_{0}$ such that

$$
\sup _{u \leq C_{0}} \sup _{\theta \in B}\left\|\frac{1}{n} \sum_{i=1}^{n}\left[W\left(u, \bar{X}_{i}^{U}(u), R_{i}\right)-\bar{W}(u ; \theta)\right] \times d_{i 0}(u)^{\prime} Y_{i}^{U}(u)-V(u ; \theta)\right\| \stackrel{p}{\rightarrow} 0
$$


The starting point is (B.8), which can, for $\theta$ in a small neighborhood of $\theta_{0}$, be rewritten as

$$
\begin{aligned}
S_{n}(\theta ; W)=\sum_{i=1}^{n} \int_{0}^{C_{i 0}}\left\{W \left(u, \bar{X}_{i}^{U}(u),\right.\right. & \left.\left.R_{i}\right)-\bar{W}(u ; \theta)\right\} \mathrm{d} N_{i}^{U}(u) \\
& +\sum_{i=1}^{n} \int_{C_{i}^{U}}^{C_{i 0}}\left\{W\left(u, \bar{X}_{i}^{U}(u), R_{i}\right)-\bar{W}(u ; \theta)\right\} \mathrm{d} N_{i}^{U}(u)
\end{aligned}
$$

Substitution of the Doob-Meier composition in the first term on the right for $N_{i}^{U}$ gives

$$
\begin{aligned}
S_{n}(\theta ; W)=\sum_{i=1}^{n} \int_{0}^{C_{i 0}}\{W(u, & \left.\left.\bar{X}_{i}^{U}(u), R_{i}\right)-\bar{W}(u ; \theta)\right\} d M_{i}^{U}(u) \\
& +\sum_{i=1}^{n} \int_{0}^{C_{i 0}}\left\{W\left(u, \bar{X}_{i}^{U}(u), R_{i}\right)-\bar{W}(u ; \theta)\right\} \kappa_{i}^{U}(u) Y_{i}^{U}(u) \mathrm{d} d u
\end{aligned}
$$

We consider both terms separately. The first term is, for $\theta$ close to $\theta_{0}$, close to $S_{n}\left(\theta_{0} ; W\right)$ and for the second term we have

$$
\left(\theta-\theta_{0}\right) \cdot \sum_{i=1}^{n} \int_{0}^{C_{i 0}}\left\{W\left(u, \bar{X}_{i}^{U}(u), R_{i}\right)-\bar{W}(u ; \theta)\right\} \times \frac{\partial \kappa_{i}^{U}(u)^{\prime}}{\partial \theta} Y_{i}^{U}(u) \mathrm{d} u+O_{p}\left(\left\|\theta-\theta_{0}\right\|^{2}\right)
$$

Returning to (C.1) we note that the second term in this equation equals

$$
\sum_{i=1}^{n}\left\{\left[W\left(C_{i 0}, \bar{X}_{i 0}\left(C_{i 0}\right), R_{i}\right)-\bar{W}\left(C_{i 0} ; \theta_{0}\right)\right] \times \theta_{0}\left(C_{i 0}\right) Y_{i}\left(C_{i 0}\right)\right\}+O_{p}\left(\left\|\theta-\theta_{0}\right\|^{2}\right)
$$

The term between brackets is the covariance between $\theta_{0}\left(C_{i 0}\right)$ and $W\left(C_{i 0}, \bar{X}_{i 0}\left(C_{i 0}\right), R_{i}\right)$ which is zero. Thus this whole term is zero for $\theta$ close to $\theta_{0}$ and we have

$$
S_{n}(\theta ; W) \approx S_{n}\left(\theta_{0} ; W\right)+n \int_{0}^{C_{0}} Z\left(u ; \theta_{0}\right) d u \cdot\left(\theta-\theta_{0}\right)
$$

Hence, approximately for the IVLR estimator $\hat{\theta}_{n}(W)$

$$
\sqrt{n}\left(\hat{\theta}_{n}(W)-\theta_{0}\right)=\left[\int_{0}^{C_{0}} Z\left(u ; \theta_{0}\right) d u\right]^{-1} \frac{1}{\sqrt{n}} S_{n}\left(\theta_{0} ; W\right)
$$

The proof of the consistency and asymptotic normality are both based upon the asymptotic linearity of $S_{n}(\theta ; W)$ in the neighborhood of the true value $\theta_{0}$. We follow the reasoning of Tsiatis (1990). Instead of a mean and variance condition, we have a mean and three covariance conditions. Let $\tilde{S}_{n}(\theta ; W)$ be the right-hand side of (C.3). The following lemma shows that the linearization in (C.3) is uniformly close to the original estimating function

Lemma 1. In neighbourhoods of $O\left(n^{-1 / 2}\right)$ of $\theta_{0}$

$$
n^{-1 / 2}\left\|\tilde{S}_{n}(\theta ; W)-S_{n}(\theta ; W)\right\|
$$

converges uniformly to zero. 
This lemma implies that $n^{-1 / 2} \tilde{S}_{n}(\theta ; W)$ and $n^{-1 / 2} S_{n}(\theta ; W)$ are asymptotically equivalent in a neighbourhood close to $\theta_{0}$.

Proof: This can be proved in lines of Tsiatis (1990) Lemma (3.1) and (3.2) and theorem (3.2) and this is, because of the analogy, not repeated here.

Proof of theorem 1 and theorem 2. According to lemma 1 are $n^{-1 / 2} S_{n}(\theta ; W)$ in a neighbourhood close to $\theta_{0}$ asymptotically equivalent to $n^{-1 / 2} \tilde{S}_{n}(\theta ; W)$. Then the estimates $\theta^{*}$ and $\hat{\theta}$, with $\tilde{S}_{n}\left(\theta^{*} ; W\right)=0$, will also be asymptotically equivalent. Clearly, $\theta^{*}$ converges in probability to $\theta_{0}$. Hence, if we show that $\sqrt{n}\left(\hat{\theta}-\theta^{*}\right) \stackrel{p}{\rightarrow} 0$ then this would imply that $\hat{\theta}$ also converges in probability to $\theta_{0}$. Tsiatis (1990) argues that lemma 1 suffices to proof this. This proves theorem 1.

According to the Mann-Wald theorem convergence in probability implies convergence in distribution. We note that $\sqrt{n}\left(\theta^{*}-\theta_{0}\right)=n^{-1 / 2} Q^{-1}(W) S_{n}\left(\theta_{0} ; W\right)$ clearly converges to a normal distribution with mean zero and variance matrix $Q^{-1}(W) \Omega(W) Q^{\prime-1}(W)$. This completes the proof of theorem 2 .

Remark. To establish detailed conditions on when $\tilde{S}_{n}(\theta ; W)$ has a unique root is rather tedious; however Ying (1993) gave an excellent general treatment on rank estimation, which can also be used for the estimating equations in this article. 


\section{Additional tables for the IVLR of reemployment bonus experiment}

Table 4: Descriptive statistics for Control,Claimant Bonus and Employer Bonus group.

\begin{tabular}{l|ccc}
\hline \hline & $\begin{array}{c}\text { Control } \\
\text { Group }\end{array}$ & $\begin{array}{c}\text { Claimant } \\
\text { bonus }\end{array}$ & $\begin{array}{c}\text { Employer } \\
\text { bonus }\end{array}$ \\
\hline White & 0.632 & 0.651 & 0.647 \\
Black & 0.271 & 0.251 & 0.256 \\
Other & 0.097 & 0.099 & 0.097 \\
Male & 0.547 & 0.563 & 0.538 \\
& & & \\
Age 20-29 & 0.425 & 0.436 & 0.424 \\
Age 30-39 & 0.333 & 0.324 & 0.326 \\
Age 40-49 & 0.179 & 0.185 & 0.187 \\
Age 50-54 & 0.063 & 0.054 & 0.064 \\
Weekly benefit & & & \\
\$51 $\$ 52-\$ 90$ & 0.088 & 0.085 & 0.084 \\
\$91-\$120 & 0.201 & 0.212 & 0.217 \\
\$121-\$160 & 0.169 & 0.176 & 0.179 \\
& 0.190 & 0.196 & 0.181 \\
Dependence allowance & 0.323 & 0.345 & 0.332 \\
Average pre-claim & 3188 & 3222 & 3215 \\
earnings & & & \\
Average age & 33.0 & 32.9 & 33.1 \\
Average weekly & 119.9 & 118.8 & 118.5 \\
N & & & \\
\hline \hline
\end{tabular}


Table 5: Instrumental Variable Linear Rank estimates for the regression coefficients of the Illinois data (Constant Bonus Effect)

\begin{tabular}{lcc|cc}
\hline \hline \multirow{2}{*}{ First stage } & \multicolumn{2}{c|}{ Claimant } & \multicolumn{2}{c}{ Employer } \\
& AFT & GAFT & AFT & GAFT \\
LNAGE & -0.5718 & -0.3424 & -0.5219 & -0.3379 \\
& $(0.0734)$ & $(0.0897)$ & $(0.0717)$ & $(0.0699)$ \\
LNBPE & 0.3528 & 0.2146 & 0.3188 & 0.2036 \\
& $(0.0510)$ & $(0.0601)$ & $(0.0512)$ & $(0.0482)$ \\
BLACK & -0.6636 & -0.3770 & -0.6264 & -0.3792 \\
& $(0.0526)$ & $(0.0842)$ & $(0.0510)$ & $(0.0641)$ \\
MALE & 0.1135 & 0.0663 & 0.0464 & 0.0295 \\
& $(0.0377)$ & $(0.0330)$ & $(0.0376)$ & $(0.0305)$ \\
LNBEN & -0.5841 & -0.3558 & -0.6263 & -0.4010 \\
& $(0.0867)$ & $(0.1011)$ & $(0.0871)$ & $(0.0865)$ \\
\hline One step & Optimal & & \multicolumn{2}{c}{} \\
& Claimant & Employer \\
& AFT & GAFT & AFT & GAFT \\
LNAGE & -0.5204 & -0.3612 & -0.4733 & -0.3110 \\
& $(0.0693)$ & $(0.0653)$ & $(0.0683)$ & $(0.0603)$ \\
LNBPE & 0.3537 & 0.2266 & 0.3133 & 0.1871 \\
& $(0.0473)$ & $(0.0449)$ & $(0.0483)$ & $(0.0424)$ \\
BLACK & -0.6162 & -0.3982 & -0.5646 & -0.3574 \\
& $(0.0509)$ & $(0.0510)$ & $(0.0495)$ & $(0.0443)$ \\
MALE & 0.1293 & 0.0691 & 0.0698 & 0.0227 \\
& $(0.0355)$ & $(0.0303)$ & $(0.0355)$ & $(0.0303)$ \\
LNBEN & -0.5924 & -0.3692 & -0.6040 & -0.3610 \\
& $(0.0813)$ & $(0.0762)$ & $(0.0826)$ & $(0.0727)$ \\
\hline \hline
\end{tabular}

${ }^{\mathrm{a}}$ GAFT piecewise constant intervals: 0-2, 2-4, 4-6, 6-10, 10-25, $25 \rightarrow$; Notes: Standard error in brackets. 
Table 6: Instrumental Variable Linear Rank estimates for the regression coefficients of the Illinois data (Time-varying Bonus effect)

\begin{tabular}{|c|c|c|c|c|}
\hline \multicolumn{5}{|c|}{ First stage } \\
\hline & \multicolumn{2}{|c|}{ Claimant } & \multicolumn{2}{|c|}{ Employer } \\
\hline & $\mathrm{AFT}$ & GAFT $^{\mathrm{a}}$ & $\mathrm{AFT}$ & GAFT $^{\mathrm{a}}$ \\
\hline \multirow[t]{2}{*}{ LNAGE } & -0.5361 & -0.3285 & -0.5233 & -0.3355 \\
\hline & $(0.0693)$ & $(0.0897)$ & $(0.0706)$ & $(0.0763)$ \\
\hline \multirow[t]{2}{*}{ LNBPE } & 0.3313 & 0.2139 & 0.3153 & 0.2029 \\
\hline & $(0.0481)$ & $(0.0617)$ & $(0.0506)$ & $(0.0530)$ \\
\hline \multirow[t]{2}{*}{ BLACK } & -0.6086 & -0.3665 & -0.6268 & -0.3771 \\
\hline & $(0.0494)$ & $(0.0861)$ & $(0.0501)$ & $(0.0740)$ \\
\hline \multirow[t]{2}{*}{ MALE } & 0.1036 & 0.0668 & 0.0461 & 0.0294 \\
\hline & $(0.0352)$ & $(0.0337)$ & $(0.0371)$ & $(0.0304)$ \\
\hline \multirow[t]{2}{*}{ LNBEN } & -0.5470 & -0.3564 & -0.6187 & -0.3989 \\
\hline & $(0.0820)$ & $(0.1043)$ & $(0.0859)$ & $(0.0959)$ \\
\hline \multicolumn{3}{|c|}{ One step Optimal } & & \\
\hline & \multicolumn{2}{|c|}{ Claimant } & \multicolumn{2}{|c|}{ Employer } \\
\hline & $\mathrm{AFT}$ & GAFT $^{\mathrm{a}}$ & $\mathrm{AFT}^{\prime}$ & GAFT $^{\mathrm{a}}$ \\
\hline \multirow[t]{2}{*}{ LNAGE } & -0.4861 & -0.3288 & -0.4529 & -0.3660 \\
\hline & $(0.0653)$ & $(0.0664)$ & $(0.0675)$ & $(0.0622)$ \\
\hline \multirow[t]{2}{*}{$\mathrm{BPE}$} & 0.3332 & 0.2061 & 0.3017 & 0.2236 \\
\hline & $(0.0442)$ & $(0.0455)$ & $(0.0474)$ & $(0.0434)$ \\
\hline \multirow[t]{2}{*}{ BLACK } & -0.5644 & -0.3615 & -0.5286 & -0.4189 \\
\hline & $(0.0476)$ & $(0.0533)$ & $(0.0488)$ & $(0.0480)$ \\
\hline \multirow[t]{2}{*}{ MALE } & 0.1176 & 0.0626 & 0.0622 & 0.0283 \\
\hline & $(0.0332)$ & $(0.0304)$ & $(0.0349)$ & $(0.0302)$ \\
\hline \multirow[t]{2}{*}{ LNBEN } & -0.5501 & -0.3343 & -0.5813 & -0.4284 \\
\hline & $(0.0765)$ & $(0.0770)$ & $(0.0815)$ & $(0.0752)$ \\
\hline
\end{tabular}

${ }^{\mathrm{a}}$ GAFT piecewise constant intervals: 0-2, 2-4, 4-6, 6-10, 10-25, $25 \rightarrow$; Notes: Standard error in brackets. 
Table 7: Estimated $\lambda$ in GAFT model for the Bonus data

\begin{tabular}{|c|c|c|c|c|}
\hline \multicolumn{5}{|c|}{ Claimant } \\
\hline & $\begin{array}{l}\text { Constant } \\
\text { first }\end{array}$ & $\begin{array}{c}\text { Bonus effect } \\
\text { opt. }\end{array}$ & $\begin{array}{l}\text { Time varying } \\
\text { first }\end{array}$ & $\begin{array}{l}\text { Bonus effect } \\
\text { opt. }\end{array}$ \\
\hline \multirow{2}{*}{$\begin{array}{l}\text { interval } \\
0-2\end{array}$} & 0.8098 & 0.7500 & 0.8625 & 0.9328 \\
\hline & $(0.4638)$ & $(0.2052)$ & $(0.5262)$ & $(0.2409)$ \\
\hline \multirow[t]{2}{*}{$2-4$} & 0.3146 & 0.2348 & 0.3542 & 0.2309 \\
\hline & $(0.3691)$ & $(0.1462)$ & $(0.4048)$ & $(0.1799)$ \\
\hline \multirow[t]{2}{*}{$4-6$} & -0.0782 & -0.0415 & -0.0390 & 0.0318 \\
\hline & $(0.2646)$ & $(0.1220)$ & $(0.3015)$ & $(0.1552)$ \\
\hline \multirow[t]{2}{*}{$6-10$} & -0.2743 & -0.1859 & -0.2341 & -0.2085 \\
\hline & $(0.2392)$ & $(0.1133)$ & $(0.2807)$ & $(0.1369)$ \\
\hline \multirow[t]{2}{*}{$10-25$} & -0.6868 & -0.6655 & -0.6077 & -0.6345 \\
\hline & $(0.1626)$ & $(0.1006)$ & $(0.1758)$ & $(0.1261)$ \\
\hline \multicolumn{5}{|c|}{ Employer } \\
\hline \multirow{3}{*}{$\begin{array}{l}\text { interval } \\
0-2\end{array}$} & $\begin{array}{l}\text { Constant } \\
\text { first }\end{array}$ & $\begin{array}{c}\text { Bonus effect } \\
\text { opt. }\end{array}$ & $\begin{array}{l}\text { Time varying } \\
\text { first }\end{array}$ & $\begin{array}{l}\text { Bonus effect } \\
\text { opt. }\end{array}$ \\
\hline & 0.7095 & 0.8929 & 0.7088 & 0.5647 \\
\hline & $(0.3063)$ & $(0.1450)$ & $(0.4375)$ & $(0.1716)$ \\
\hline \multirow[t]{2}{*}{$2-4$} & 0.2540 & 0.4451 & 0.2542 & 0.1464 \\
\hline & $(0.2134)$ & $(0.0939)$ & $(0.3344)$ & $(0.1227)$ \\
\hline \multirow[t]{2}{*}{$4-6$} & -0.1217 & -0.1178 & -0.1195 & 0.0875 \\
\hline & $(0.2008)$ & $(0.0925)$ & $(0.2330)$ & $(0.1050)$ \\
\hline \multirow[t]{2}{*}{$6-10$} & -0.4552 & -0.2707 & -0.4526 & -0.4098 \\
\hline & $(0.1516)$ & $(0.0751)$ & $(0.2255)$ & $(0.0975)$ \\
\hline \multirow[t]{2}{*}{$10-25$} & -0.7492 & -0.6826 & -0.7180 & -0.6057 \\
\hline & $(0.0971)$ & $(0.0372)$ & $(0.1015)$ & $(0.0491)$ \\
\hline
\end{tabular}

Notes: Standard error in brackets. 